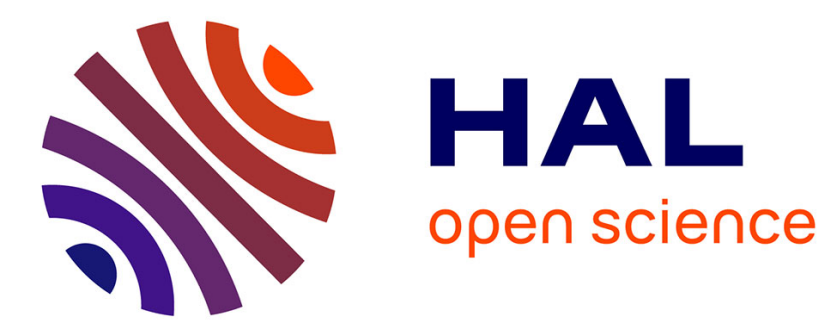

\title{
Half-explicit timestepping schemes on velocity level based on time-discontinuous Galerkin methods
}

Thorsten Schindler, Shahed Rezaei, Jochen Kursawe, Vincent Acary

\section{To cite this version:}

Thorsten Schindler, Shahed Rezaei, Jochen Kursawe, Vincent Acary. Half-explicit timestepping schemes on velocity level based on time-discontinuous Galerkin methods. Computer Methods in Applied Mechanics and Engineering, 2015, 290, pp.250-276. 10.1016/j.cma.2015.03.001 . hal-01235242

\section{HAL Id: hal-01235242 \\ https://inria.hal.science/hal-01235242}

Submitted on 29 Nov 2015

HAL is a multi-disciplinary open access archive for the deposit and dissemination of scientific research documents, whether they are published or not. The documents may come from teaching and research institutions in France or abroad, or from public or private research centers.
L'archive ouverte pluridisciplinaire HAL, est destinée au dépôt et à la diffusion de documents scientifiques de niveau recherche, publiés ou non, émanant des établissements d'enseignement et de recherche français ou étrangers, des laboratoires publics ou privés. 


\title{
Half-explicit timestepping schemes on velocity level based on time-discontinuous Galerkin methods
}

\author{
Thorsten Schindler ${ }^{\mathrm{a}, *}$, Shahed Rezaei ${ }^{\mathrm{b}}$, Jochen Kursawe ${ }^{\mathrm{c}}$, Vincent Acary ${ }^{\mathrm{d}}$ \\ ${ }^{a}$ Technische Universität München, Institute of Applied Mechanics, Boltzmannstraße 15, 85748 Garching, Germany \\ ${ }^{b}$ RWTH Aachen, Institute of Applied Mechanics, Mies-van-der-Rohe-Str. 1, 52074 Aachen, Germany \\ ${ }^{c}$ Mathematical Institute, University of Oxford, Andrew Wiles Building, Radcliffe Observatory Quarter, Woodstock Road, Oxford OX2 6GG, United \\ Kingdom \\ dINRIA Rhône-Alpes, Centre de recherche Grenoble, 655 avenue de l'Europe, Inovallée de Montbonnot, 38334 St Ismier Cedex, France
}

\begin{abstract}
This paper presents a time-discretization scheme for the simulation of nonsmooth mechanical systems. These consist of rigid and flexible bodies, joints as well as contacts and impacts with dry friction. The benefit of the proposed formalism is both the consistent treatment of velocity jumps, e.g. due to impacts, and the automatic local order elevation in non-impulsive intervals at the same time. For an appropriate treatment of constraints in impulsive and non-impulsive intervals, constraints are implicitly formulated on velocity level in terms of an augmented Lagrangian technique [1]. They are satisfied exactly without any penetration. For efficiency reasons, all other evaluations are explicit which yields a half-explicit method [2-8].

The numerical scheme is an extended timestepping scheme for nonsmooth dynamics according to Moreau [9]. It is based on time-discontinuous Galerkin methods to carry over higher order trial functions of event-driven integration schemes to consistent timestepping schemes for nonsmooth dynamical systems with friction and impacts. Splitting separates the portion of impulsive contact forces from the portion of non-impulsive contact forces. Impacts are included within the discontinuity of the piecewise continuous trial functions, i.e., with first-order accuracy. Nonimpulsive contact forces are integrated with respect to the local order of the trial functions. In order to satisfy the constraints, a set of nonsmooth equations has to be solved in each time step depending on the number of stages; the solution of the velocity jump together with the corresponding impulse yields another nonsmooth equation. All nonsmooth equations are treated separately by semi-smooth Newton methods.

The integration scheme on acceleration level was first introduced in [10] labeled "forecasting trapezoidal rule". It was analyzed and applied to a decoupled bouncing ball example concerning principal suitability without taking friction into account. In this work, the approach is algorithmically specified, improved and applied to nonlinear multicontact examples with friction. It is compared to other numerical schemes and it is shown that the newly proposed integration scheme yields a unified behavior for the description of contact mechanical problems.
\end{abstract}

Keywords: timestepping scheme, discontinuous Galerkin method, nonsmooth dynamics, flexible multibody system, friction, impact, index reduction

\section{Introduction}

In this paper, we study numerical integration schemes for the simulation of nonsmooth mechanical systems. Rigid and flexible bodies, joints as well as contacts and impacts with dry friction constitute the mechanical models. Thereby, we formulate the contact conditions as constraints and do not allow any penetration, e.g. due to penalty techniques. As a result, velocity jumps occur during the transient simulation of semi-discrete models and we have to be cautious in the formulation of efficient and stable time-discretization schemes.

\footnotetext{
* Corresponding author

Email addresses: thorsten. schindler@mytum. de (Thorsten Schindler), shahed.rezaei@rwth-aachen. de (Shahed Rezaei), Jochen.Kursawe@maths.ox.ac.uk (Jochen Kursawe), vincent.acary@inria.fr(Vincent Acary)
} 
We distinguish two cases.

1. non-impulsive contact forces - For the contact between flexible bodies, the contact force is finite in continuum models, i.e., in not semi-discrete models, although velocity jumps may arise. Classic (implicit) time integration schemes for computational mechanical problems, i.e., members of the Newmark family [11-15], have been adapted to these demands and extended with respect to contact/velocity updates (Laursen-Love scheme) [16, 17]. Another strategy to preferably get a well-posed problem is the application of energy-momentum paradigms like in energy-momentum schemes $[15,18]$, i.e., modifications of the midpoint rule [14], to impact problems (Laursen-Chawla scheme) [16]. A contact-stabilized Newmark scheme is proposed in [19].

2. impulsive contact forces - For the contact between rigid bodies, the reaction forces are impulsive and the classic time integration schemes do not work anymore [20]. The application of mass redistribution techniques [17] is a procedure, which reminds of penalty approaches with the benefit of having a theoretical foundation [21]. However in [22], it is shown that all these schemes suffer from oscillations in the relative contact velocities. Event-driven schemes and timestepping schemes are further concepts to simulate rigid multibody systems or semi-discrete systems consistently by applying impact laws. Thereby, event-driven schemes resolve impact events to a high precision. In-between impact events, standard integration schemes are used. Classic timestepping schemes do not resolve impact events, but include their possible existence directly in the discretization. Thus, they have low accuracy in non-impulsive intervals, whereas event-driven schemes may get inefficient and inconsistent for many impact events [20].

The aim of the present paper is to improve the consistent and robust concept of timestepping schemes for semidiscrete mechanical systems. The main drawback is the lack of problem adaptive accuracy in non-impulsive intervals. Classic timestepping schemes can be embedded within the context of time-discontinuous Galerkin methods, when we choose piecewise constant trial functions for the velocity approximation [10]. In the aforementioned paper, two different families of timestepping schemes on acceleration level based on discontinuous Galerkin methods have been introduced and analyzed. They differ in the interpretation, if one assumes the velocity jump at the beginning, called $D^{+}$timestepping schemes, or at the end, called $D^{-}$timestepping schemes, of each discretization interval. First, using higher-order but piecewise continuous trial functions for the velocity, and, second, splitting of impulsive and nonimpulsive contact reactions, offer the opportunity to both stay consistent and benefit from a higher-order integration of non-impulsive contact reactions. The "forecasting trapezoidal rule" is the $D^{-}$representative for piecewise linear velocity trial functions; concerning function evaluations and implementation complexity, it is the easiest scheme. Its basic practical applicability apart from theoretical propositions has been shown with a decoupled bouncing ball example. It is the basis for extensions in the present paper. We algorithmically specify and improve it and apply it to nonlinear multi-contact examples with friction. Thereby for an automatic switching between non-impulsive and impulsive intervals, constraint equations for non-impulsive reactions are also formulated on velocity level in an augmented Lagrangian setting [1] like the constraint equations for contact impulse and jumping velocity. Therefore, we focus on half-explicit methods and evaluate all other magnitudes but the constraints explicitly [2-8]. The number of nonsmooth equations for the constraints depends on the number of stages, i.e., the local order of the underlying trial functions. These three nonsmooth equations are solved separately by semi-smooth Newton methods [1, 23, 24].

The recent proposal for higher-order integration of non-impulsive contact reactions on basis of the generalized- $\alpha$ scheme [25] is also footing on a specific splitting approach. Splitting is interpreted as separating the "flow" of applied forces, active contact reactions and non-active contact reactions in the classic "Strang-sense" [26]. In contrast, we split non-impulsive and impulsive reactions directly.

The paper introduces the new time-discretization scheme step-by-step. The proposed (intermediate) schemes are directly evaluated using an impacting slider-crank mechanism which we introduce in Sect. 2.1. The paper is organized as follows. Section 2 introduces the equations of motion and common time-discretization schemes for nonsmooth mechanical systems. Half-explicit timestepping schemes on acceleration level are introduced in Sect. 3. We generalize the approach in [10] to the multi-contact case and propose a first well working timestepping scheme, which performs automatically with higher order if there are no impacts but is consistent for impulsive periods, as well (Table 3). Due to the acceleration level approach, contact forces are calculated in a natural way. However, we show that drift-off effects may occur. That is why, we propose also a second timestepping scheme. The final half-explicit scheme on velocity level is derived and described in Sect. 4 (Table 6) as well as tested within the example of the slider-crank mechanism. It is embedded in the notation of [2] in Appendix A and in Appendix B. In Sect. 5, we 
analyze the performance of our proposed timestepping schemes with the example of the slider-crank mechanism and a bouncing ball example. In Sect. 6, we extend this analysis to flexible systems with the examples of an elastic bar and a rotor. Section 7 concludes the paper. For a compact representation of the present paper, we refer to the longer technical report [27] for further details.

\section{Equations of motion and time-discretization}

This section introduces the continuous equations of motion, the impacting slider-crank mechanism as an accompanying example and basic time-discretization schemes. We consider an impacting mechanical system with dry friction. For non-impulsive motion in time, it is described by the following set of equations

$$
\dot{q}=v,
$$

and

$$
\begin{aligned}
M \dot{v} & =h+W_{N} \lambda_{N}+W_{T} \lambda_{T}, \\
& \leq \leq g_{N} \perp \lambda_{N} \geq 0, \\
& \left\{\begin{array}{ll}
\left\|\lambda_{T}\right\| \leq \mu \lambda_{N} & \text { for } \dot{g}_{T}=0 \wedge g_{N} \leq 0 \\
\lambda_{T}=-\frac{\dot{g}_{T}}{\left\|\dot{s}_{T}\right\|} \mu \lambda_{N} & \text { for } \dot{g}_{T} \neq 0 \wedge g_{N} \leq 0
\end{array} .\right.
\end{aligned}
$$

For mechanical systems, these equations typically occur after semi-discretization in space of respective mathematical models, e.g. by the finite element method [17]. If one does not use any classic concept of regularization by introducing contact stiffnesses or contact potentials [28,29], an impact may occur at time $t_{j} \geq 0$ and we have to use

$$
\begin{aligned}
M\left(q\left(t_{j}\right)\right)\left(v_{j}^{+}-v_{j}^{-}\right) & =W_{N}\left(q\left(t_{j}\right)\right) \Lambda_{N, j}+W_{T}\left(q\left(t_{j}\right)\right) \Lambda_{T, j}, \\
& 0 \leq \dot{g}_{N, j}^{+}+\varepsilon_{N} \dot{g}_{N, j}^{-} \perp \Lambda_{N, j} \geq 0, \\
& \begin{cases}\left\|\Lambda_{T, j}\right\| \leq \mu \Lambda_{N, j} & \text { for } \dot{g}_{T, j}^{+}+\varepsilon_{T} \dot{g}_{T, j}^{-}=0 \\
\Lambda_{T, j}=-\frac{\dot{g}_{T, j}^{+}+\varepsilon_{T} \dot{g}_{T, j}^{-}}{\left\|\dot{g}_{T, j}^{+}+\varepsilon_{T} \dot{g}_{T, j}^{-}\right\|} \mu \Lambda_{N, j} & \text { for } \dot{g}_{T, j}^{+}+\varepsilon_{T} \dot{g}_{T, j}^{-} \neq 0\end{cases}
\end{aligned}
$$

instead of (2)-(4).

We want to calculate the generalized position and generalized velocity functions on the time interval $I:=[0, T]$ with $T>0$ :

$$
\begin{aligned}
& q:[0, T] \rightarrow \mathbb{R}^{N_{d}}, t \mapsto q(t), \\
& v:[0, T] \rightarrow \mathbb{R}^{N_{d}}, t \mapsto v(t) .
\end{aligned}
$$

With the initial conditions

$$
\begin{aligned}
& q(0)=q_{0}, \\
& v(0)=v_{0},
\end{aligned}
$$

the evolution of $q$ and $v$ is non-impulsive almost everywhere due to (2)-(4).

The generalized mass matrix satisfies

$$
M: \mathbb{R}^{N_{d}} \rightarrow \mathbb{R}^{N_{d}, N_{d}}, q \mapsto M(q)
$$

and the nonlinear generalized force has the structure

$$
h: \mathbb{R}^{N_{d}} \times \mathbb{R}^{N_{d}} \rightarrow \mathbb{R}^{N_{d}},(q, v) \mapsto h(q, v) .
$$


The matrices of generalized force directions $W_{N}$ and $W_{T}$ are the derivatives of the nonlinear normal and tangential gap functions

$$
\begin{aligned}
& g_{N}: \mathbb{R}^{N_{d}} \rightarrow \mathbb{R}^{N_{c}}, q \mapsto g_{N}(q), \\
& g_{T}: \mathbb{R}^{N_{d}} \rightarrow \mathbb{R}^{2 N_{c}}, q \mapsto g_{T}(q)
\end{aligned}
$$

with respect to $q$. These describe normal distances and tangential displacements of contacting bodies in a mechanical system and are therefore constraining its motion. The Lagrange multipliers

$$
\lambda_{N}:[0, T] \rightarrow \mathbb{R}^{N_{c}}, t \mapsto \lambda_{N}(t)
$$

can be identified with normal contact forces in the equations of motion (2) and in the non-penetration condition (3). The Lagrange multipliers

$$
\lambda_{T}:[0, T] \rightarrow \mathbb{R}^{2 N_{c}}, t \mapsto \lambda_{T}(t)
$$

can be identified with tangential contact forces in the equations of motion (2) and in (4), which contains sticking and sliding conditions with the friction coefficient $\mu \geq 0$ and the time-derivative denoted by a dot. The index set $\mathscr{I}_{0}$ contains all constraints on the system.

$$
\begin{aligned}
\mathscr{I}_{1}^{q} & =\left\{k \in \mathscr{I}_{0}: g_{N_{k}}(q) \leq 0\right\} \text { is the continuous index set of the closed constraints, } \\
\mathscr{I}_{2}^{q, v \pm} & =\left\{k \in \mathscr{I}_{1}^{q}: \dot{g}_{N_{k}}\left(q, v^{ \pm}\right) \leq 0\right\} \text { is the continuous index set of the closed constraints, which stay closed }
\end{aligned}
$$

For countable time instances $t_{j}$, the evolution of the mechanical system might get impulsive, e.g. if $g_{N_{k^{*}}}\left(q\left(t_{j}\right)\right)=0$ for some component $k^{*}$, but $g_{N_{k^{*}}}(q(t))>0$ for $t_{j}-\delta \leq t<t_{j}$ with an appropriate $\delta>0$. The impact equations (5), Newton's impact law (6) with the coefficient of restitution $\varepsilon_{N} \in[0,1]$ and Newton's impact law (7) with the coefficient of restitution $\varepsilon_{T} \in[0,1]$ have to be solved instead of (2)-(4). This possibly leads to jumps in the velocity variables. Their derivatives do not exist anymore in the classical sense. One has to define the left-hand and right-hand limits

$$
\begin{array}{ll}
\dot{g}_{N_{j}}^{-}:=\lim _{t \uparrow t_{j}} \dot{g}_{N}(t), \quad \dot{g}_{T_{j}}^{-}:=\lim _{t \uparrow t_{j}} \dot{g}_{T}(t), \\
\dot{g}_{N_{j}}^{+}:=\lim _{t \downarrow t_{j}} \dot{g}_{N}(t), & \dot{g}_{T_{j}}^{+}:=\lim _{t \downarrow t_{j}} \dot{g}_{T}(t) .
\end{array}
$$

Then, the Lagrange multipliers

$$
\Lambda_{N_{j}}=\lim _{\delta \downarrow 0} \int_{t_{j}-\delta}^{t_{j}} \lambda_{N} \mathrm{~d} t, \quad \Lambda_{T_{j}}=\lim _{\delta \downarrow 0} \int_{t_{j}-\delta}^{t_{j}} \lambda_{T} \mathrm{~d} t
$$

describe the finite impulsive interaction in the sense of distributions.

Equations (1)-(7) describe impacting mechanical systems in general. Their mechanical, mathematical and numerical behaviour have been detailed in the monographs [20, 30-35] even discussing more sophisticated impact laws of kinetic or energetic type.

\subsection{Slider-crank mechanism}

The slider-crank mechanism in Fig. 1 is a nonlinear benchmark example for an impacting mechanical system with dry friction. Considering the geometry of the slider, the nonlinear normal and tangential gap functions split up for each corner. Fixed characteristics used in this work are given in Table 1; further information can be found in the report [27]. We focus on numerical integration and therefore point out particular previous work just in this direction. Classically, we distinguish event-driven schemes, which are also called event-tracking schemes, and timestepping schemes, which are also known as event-capturing schemes. 


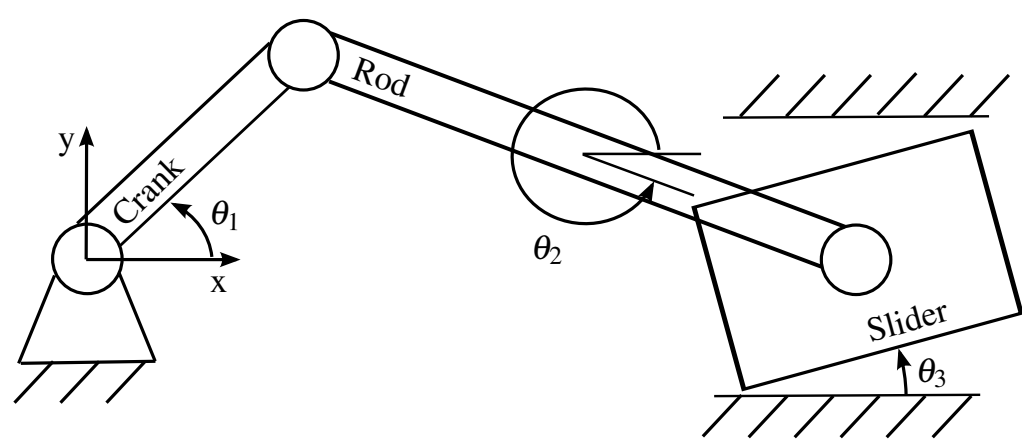

Figure 1: Slider-crank mechanism with unilateral constraints and friction [36, 37].

\subsection{Event-driven schemes}

An event-driven scheme discretizes the non-impulsive equations (1)-(4) by a classic numerical rule [20, Chap. 8]. At the same time, it observes the gap functions for open $\left(g_{N}>0\right)$-close $\left(g_{N} \leq 0\right)$ transitions or stick $\left(\dot{g}_{T}=0\right)$-slip $\left(\dot{g}_{T} \neq 0\right)$ transitions. In such cases, the exact transition time is resolved by a root-finding algorithm with respect to a given tolerance because the structure, i.e., the actual degree of freedom of the mathematical model, changes and therefore also the numerical discretization has to be adapted. In the case of an open-close transition, the impact equations (5)-(7) have to be solved separately and subsequently the non-impulsive integration has to be restarted. LSODAR $^{1}$ for index 0 or DASKR ${ }^{2}$ for index 2 formulations are typical examples for the derivation of the numerical model by standard integration schemes. Thereby, the relation of the index of the non-impulsive equations (1)-(4) and of their numerical counterpart play a crucial part for the discretization [8, 38]. We discuss this concept for timestepping schemes in the following subsection. As event-driven schemes resolve the exact transition times, they, first, cannot resolve Zeno phenomena, i.e., an infinite number of impacts in a finite time interval. Second, even the resolution of the transition time itself may be crucial as the used tolerance is depending on the specific mechanical problem, e.g. on penetration velocities. These are two main drawbacks of event-driven schemes for their application to the mathematical description of impacting mechanical systems. However for non-impulsive intervals, event-driven schemes are extremely efficient because they are based on sophisticated numerical integration schemes with e.g. high accuracy for the numerical representation of the non-impulsive equations.

\subsection{Classic timestepping schemes}

Classic timestepping schemes do not distinguish impulsive and non-impulsive equations, i.e., they do not resolve exact transition times. Hence, no additional tolerances are required. Classic timestepping schemes discretize the equations of motion including the constraints, impact laws and impact equations in a physically consistent and uniform way [20, Chap. 10]. This means that possible impacts are the determining factor for the order of classic timestepping schemes. In comparison to the maximum possible order in non-impulsive phases, the actual order may be low. However, convergence results are available for classic timestepping schemes and not for event-driven schemes. Two mainstream concepts for classic timestepping schemes exist: Schatzman-Paoli $[39,40]$ and Moreau-Jean schemes [9, 41]. The Schatzman-Paoli scheme discretizes normal gap functions on the level of positions and satisfies impact laws after several time steps. That is why, we prefer to formulate the impact law on the level of velocities in discrete time, which is the core of Moreau-Jean schemes.

To exemplary formulate the classic explicit Moreau-Jean scheme on an arbitrary time interval $I_{i}:=\left[t_{i-1}, t_{i}\right)$, we

\footnotetext{
${ }^{1}$ http://www.netlib.org/odepack/

${ }^{2}$ http://www.netlib.org/ode/
} 


\begin{tabular}{ll}
\hline Geometrical characteristics & $l_{1}=0.1530 \mathrm{~m}$ (length crank) \\
& $l_{2}=0.3060 \mathrm{~m}$ (length rod) \\
& $a=0.0500 \mathrm{~m}$ (half-length slider) \\
& $b=0.0250 \mathrm{~m}$ (half-height slider) \\
& $c=0.0010 \mathrm{~m}$ (gap of the notch) \\
\hline Inertia properties & $m_{1}=0.0380 \mathrm{~kg}$ \\
& $m_{2}=0.0380 \mathrm{~kg}$ \\
& $m_{3}=0.0760 \mathrm{~kg}$ \\
& $J_{1}=7.4 \cdot 10^{-5} \mathrm{kgm}^{2}$ \\
& $J_{2}=5.9 \cdot 10^{-4} \mathrm{kgm}^{2}$ \\
& $J_{3}=2.7 \cdot 10^{-6} \mathrm{kgm}^{2}$ \\
\hline Force elements & $\gamma=9.81 \mathrm{~m} / \mathrm{s}^{2}$ \\
\hline Contact parameters & $\varepsilon_{N_{1}}=\varepsilon_{N_{2}}=\varepsilon_{N_{3}}=\varepsilon_{N_{4}}=0.4$ \\
& $\varepsilon_{T_{1}}=\varepsilon_{T_{2}}=\varepsilon_{T_{3}}=\varepsilon_{T_{4}}=0.0$ \\
& $\mu_{1}=\mu_{2}=\mu_{3}=\mu_{4}=0.01$ \\
\hline Initial conditions & $\theta_{10}=0.0$ \\
& $\theta_{2_{0}}=0.0$ \\
& $\theta_{3_{0}}=0.0$ \\
& $\omega_{1_{0}}=150.01 / \mathrm{s}$ \\
& $\omega_{2_{0}}=-75.01 / \mathrm{s}$ \\
& $\omega_{3_{0}}=0.01 / \mathrm{s}$ \\
\hline
\end{tabular}

Table 1: Characteristics of the slider-crank mechanism with unilateral constraints and friction [36, 37].

construct the approximations at time $t_{i}$

$$
\begin{aligned}
q_{i} & \approx q\left(t_{i}\right), \\
v_{i}^{-} & \approx \lim _{t \uparrow t_{i}} v(t), \quad v_{i}^{+} \approx \lim _{t \downarrow t_{i}} v(t), \\
\Lambda_{N_{i}} & \approx \sum_{j: t_{j} \in I_{i}} \Lambda_{N, j}, \quad \Lambda_{T_{i}} \approx \sum_{j: t_{j} \in I_{i}} \Lambda_{T, j}
\end{aligned}
$$

and use them in the abbreviations

$$
\begin{aligned}
& M_{i}^{-1}:=M^{-1}\left(q_{i}\right), \\
& h_{i}^{ \pm}:=h\left(q_{i}, v_{i}^{ \pm}\right), \\
& W_{N_{i}}:=W_{N}\left(q_{i}\right), W_{T_{i}}:=W_{T}\left(q_{i}\right) .
\end{aligned}
$$

Then, the classic explicit Moreau-Jean scheme with the time step-size $\Delta t_{i}:=t_{i}-t_{i-1}$ is given by

$$
\begin{aligned}
q_{i} & =q_{i-1}+\Delta t_{i} v_{i-1}^{+} \\
v_{i}^{+} & =v_{i-1}^{+}+\Delta t_{i} M_{i-1}^{-1} h_{i-1}^{+}+M_{i}^{-1}\left[W_{N_{i}} \Lambda_{N_{i}}+W_{T_{i}} \Lambda_{T_{i}}\right]
\end{aligned}
$$

together with the active normal impact equations on velocity level

$$
\Lambda_{N_{i}, \mathscr{G}_{1, M}^{i}}-\operatorname{proj}_{\mathbb{R}_{0}^{+}}\left(\Lambda_{N_{i}, \mathscr{I}_{1, M}^{i}}-r\left(\dot{g}_{N_{i}, \mathscr{I}_{1, M}^{i}}^{+}+\varepsilon_{N} \dot{g}_{N_{i-1}, \mathscr{I}_{1, M}^{i}}^{+}\right)\right)=0
$$

and the active tangential impact equations on velocity level

$$
\Lambda_{T_{i}, \mathscr{I}_{1, M}^{i}}-\operatorname{proj}_{C_{T}\left(\Lambda_{N_{i}, \mathscr{Y}_{1, M}^{i}}^{i}\right)}\left(\Lambda_{T_{i}, \mathscr{I}_{1, M}^{i}}-r\left(\dot{g}_{T_{i}, \mathscr{I}_{1, M}^{i}}^{+}+\varepsilon_{T} \dot{g}_{T_{i-1}, \mathscr{I}_{1, M}^{i}}^{+}\right)\right)=0
$$


where $r$ is a stabilization parameter, see below. To reduce the degree of nonlinearity, usually one estimates active contact equations in advance by checking if a prediction, e.g. an explicit Euler step with time step-size $\Delta t / 2$, is negative:

$$
\mathscr{I}_{1, M}^{i}:=\left\{k \in \mathscr{I}_{0}: \tilde{g}_{N_{k}}^{i}:=g_{N_{k}}\left(q_{i-1}\right)+\frac{\Delta t_{i}}{2} \dot{g}_{N k}\left(q_{i-1}, v_{i-1}^{+}\right) \leq 0\right\} .
$$

We have used the projection to a convex set $C$ [42] to equivalently transform complementarity formulations for normal and tangential contacts. It has to be interpreted row-by-row [43]:

$$
\operatorname{proj}_{C}: \mathbb{R} \rightarrow \mathbb{R}, x \mapsto \operatorname{proj}_{C}(x)=\underset{x^{*} \in C}{\arg \min }\left\|x-x^{*}\right\| .
$$

The convex set to describe dry friction is the friction disc with the local normal force as radius:

$$
C_{T}: \mathbb{R} \rightarrow \mathscr{P}\left(\mathbb{R}^{2}\right): y \mapsto C_{T}(y)=\left\{x \in \mathbb{R}^{2}|\|x\| \leq \mu| y \mid\right\} .
$$

The parameter $r>0$ is mathematically arbitrary. It physically transforms units and can also be interpreted as a stabilization in an augmented Lagrangian setting [1]. Therefore, it can be used to improve convergence of numerical solution schemes, which are applied to solve (29)-(32) for the unknowns $q_{i}, v_{i}^{+}, \Lambda_{N_{i}, \mathscr{I}_{1, M}^{i}}$ and $\Lambda_{T_{i}, \mathscr{I}_{1, M}^{i}}$. The classic explicit Moreau-Jean scheme does not adapt the time step-size and naturally combines non-impulsive and impulsive equations, contacts as well as impacts by calculating implicitly the finite mean impulses $\Lambda_{N_{i}, \mathscr{J}_{1, M}^{i}}$ and $\Lambda_{T_{i}, \mathscr{J}_{1, M}^{i}}$ within $I_{i}$; their trajectory within $I_{i}$ in this sense is an assumption. Hence, several extensions are of possible interest and are current research topics:

- Constraint stabilization $[25,37,44]$

Moreau-Jean schemes in general are formulated on the level of velocities. Hence, a linear drift from the non-penetration invariant will occur [45, 46]. The Gear-Gupta-Leimkuhler formulation [47] treats the nonpenetration invariant as additional constraint and therefore inserts a derivative projection; applied to Moreau's timestepping interpretation of the midpoint rule [9], this can be understood as a symmetric projection [14, 48].

- High-frequency damping $[25,49,50]$

Artificial high-frequency oscillations occur due to finite element discretizations and e.g. excitation in contact problems. One possible remedy is applying the generalized- $\alpha$ method [13] in the sense of timestepping schemes.

- Variational integration [51]

Sophisticated integration schemes can often be derived from a discrete variational principle [52]. This concept is tried to be extended to timestepping schemes for impacting mechanical systems. However, some kind of splitting seems to be necessary because a symplectic method will not stay symplectic for an impact event [53].

- Higher order timestepping and step-size adaptation

There are two types of higher order timestepping schemes which can consistently deal with impacts.

- Augmented timestepping schemes [54, 55]

Within an augmented timestepping scheme, one applies classic augmentation strategies like order extrapolation whenever one heuristically expects no impacts during an integration step. Extrapolation induces instabilities in closed contacts because of chattering in Aitken-Neville schemes or because of the absence of splitting of non-impulsive and impulsive force propagations. It is a serial process. That is why, usually one applies a fixed increased order when we anticipate a non-impulsive phase. Also from a practical point of view, i.e., not infinitely-differentiable input data, the application of an extrapolation-based augmentation strategy like in [56] seems to be over-engineered.

- Mixed timestepping schemes [10, 57, 58]

A mixed timestepping scheme couples standard integration methods for non-impulsive differential algebraic equations with timestepping schemes for impulsive phases usually by heuristics. 
We extend [10] to multi-contact problems with friction. In [10], heuristics for switching between impulsive and non-impulsive phases can be avoided by embedding in discontinuous Galerkin methods. The synchronization of fixed increased order integration and consistent low order integration is automatic but as we will see perhaps not the most robust way for an implementation.

Step size adaptation can be applied for both higher order timestepping strategies. It extends the classic approach $[8,59]$ by heuristics which meet the timestepping idea. We mention foreseeing gap-estimations, retrospective time-step bisection, time-step size switching maintaining the overall integration order and error estimation based on Richardson extrapolation. For the latter, one could exclude velocities, discuss appropriate norms, separate impacts interval-by-interval or include the penetration depth of closed contacts. However to the knowledge of the authors, these strategies have not been theoretically analyzed in the literature.

\subsection{Calculation of contact forces on acceleration level}

On the one hand, impulses are naturally calculated on velocity level like in Moreau-Jean schemes, on the other hand, contact forces are naturally calculated on acceleration level. We explain the computation for normal contact forces; frictional contact forces are treated similarly. Thereby, it seems not to be a bad idea to use (3) together with (2) for the calculation of a contact force $\lambda_{N}$ at a certain time also for more general nonlinear mechanical systems. We interpret (3) on acceleration level

$$
\lambda_{N}= \begin{cases}0 & \text { if } g_{N}>0 \vee \dot{g}_{N}>0 \\ \operatorname{proj}_{\mathbb{R}_{0}^{+}}\left[\lambda_{N}-r \ddot{g}_{N}\right] & \text { else }\end{cases}
$$

using the projection-formulation row-by-row. Often, we have to deal with holonomic-scleronomic constraints:

$$
\begin{aligned}
& \dot{g}_{N}=W_{N}^{T} v, \\
& \ddot{g}_{N}=\underbrace{\dot{W}_{N}^{T}}_{\sum_{i} \frac{\partial W_{N}^{T}}{\partial q_{i}} v_{i}} v+W_{N}^{T} \dot{v} .
\end{aligned}
$$

We can simplify (38) to

$$
\ddot{g}_{N}=Q_{N} v \mathbf{\square}+W_{N}^{T} \dot{v}
$$

with $v_{\text {m }}$ being a vector containing squared generalized velocity combinations and $Q_{N}$ denoting a respective transformation matrix. In comparison to the decoupled bouncing ball example, the nonlinearity in (39) and in the right-hand side $h$ arises in general. We eliminate $\dot{v}$ in (39) by using (2):

$$
\ddot{g}_{N}=Q_{N} v_{\boldsymbol{\square}}+W_{N}^{T} M^{-1} h+G_{N} \lambda_{N}
$$

with the Delassus matrix [32], i.e., the mass action matrix [33],

$$
G_{N}=W_{N}^{T} M^{-1} W_{N}
$$

We focus on active contacts, $\mathscr{I}_{2}$ on acceleration level, and transform (36) formally using row-by-row interpretation:

$$
\lambda_{N, \mathscr{I}_{2}}=\operatorname{proj}_{\mathbb{R}_{0}^{+}}\left[\lambda_{N, \mathscr{I}_{2}}-r(\underbrace{Q_{N, \mathscr{I}_{2}} v_{\mathbf{\square}}+W_{N, \mathscr{I}_{2}}^{T} M^{-1} h+G_{N, \mathscr{I}_{2}} \lambda_{N, \mathscr{I}_{2}}}_{\ddot{g}_{N, \mathscr{I}_{2}}})\right] .
$$

In the multi-contact case, active contacts might be depending. Hence if we decide to use e.g. a semi-smooth Newton method as root-finding algorithm to solve (42), we have to switch to semi-smooth variants of the Gauss-Newton method. We use a fixed nominal parameter value $r=0.1$ [43]. 


\section{Half-explicit timestepping schemes on acceleration level}

This section generalizes timestepping schemes based on discontinuous Galerkin methods [10] to multi-contact examples and shows their advantages and disadvantages. The theoretical benefit of these schemes over mixed timestepping schemes is that they do not rely on heuristics. Thereby as in [10], we start by introducing contact forces on acceleration level and by splitting non-impulsive and impulsive force propagation. We try to get some experience with linear trial functions for the velocity discretization, i.e., half-explicit "forecasting" trapezoidal rules, not regarding friction:

$$
\begin{aligned}
q_{i-1,0} & =q_{i-1}, \\
q_{i-1,1} & =q_{i-1}+\frac{\Delta t_{i}}{2}\left\{v_{i-1,0}+v_{i-1,1}\right\}, \\
q_{i} & =q_{i-1}+\frac{\Delta t_{i}}{2}\left\{v_{i-1,0}+v_{i-1,1}\right\}, \\
v_{i-1,0} & =v_{i-1}^{+}, \\
v_{i-1,1} & =v_{i-1}^{+}+\Delta t_{i} M_{i-1}^{-1}\left[h_{i-1}^{+}+W_{N_{i-1}} \lambda_{N_{i-1}}^{+}\right], \\
v_{i}^{+} & =v_{i-1}^{+}+\frac{\Delta t_{i}}{2} M_{i-1}^{-1}\left[h_{i-1}^{+}+W_{N_{i-1}} \lambda_{N_{i-1}}^{+}\right]+\frac{\Delta t_{i}}{2} M_{i-1,1}^{-1}\left[h_{i-1,1}+W_{N_{i-1,1}} \lambda_{N_{i}}^{-}\right]+M_{i}^{-1} W_{N_{i}} \Lambda_{N_{i}}
\end{aligned}
$$

together with the active contact equations on acceleration level

$$
\begin{aligned}
\lambda_{N_{i-1}, \mathscr{I}_{2}^{(i-1), 0}}^{+}-\operatorname{proj}_{\mathbb{R}_{0}^{+}}\left[\lambda_{N_{i-1}, \mathscr{I}_{2}^{(i-1), 0}}^{+}-r \ddot{g}_{N_{i-1}^{+}, \mathscr{I}_{2}^{(i-1), 0}}^{+}\right. & =0, \\
\lambda_{N_{i}, \mathscr{I}_{2}^{(i-1), 1}}^{-}-\operatorname{proj}_{\mathbb{R}_{0}^{+}}\left[\lambda_{N_{i}, \mathscr{I}_{2}^{(i-1), 1}}^{-}-r_{N_{i}, \mathscr{I}_{2}^{(i-1), 1}}^{-}\right] & =0
\end{aligned}
$$

and the active impact equations on velocity level similar to (31) but referring to another index set, which we have to define. It is

$$
\begin{aligned}
\mathscr{I}_{1}^{i, l} & =\left\{k \in \mathscr{I}_{0}: g_{N_{k}}\left(q_{i, l}\right) \leq 0\right\} \text { the discrete index set of the closed constraints, } \\
\mathscr{I}_{2}^{i, l \pm} & =\left\{k \in \mathscr{I}_{1}^{i, l}: \dot{g}_{N_{k}}\left(q_{i, l}, v_{i, l}^{ \pm}\right) \leq 0\right\} \text { the discrete index set of the closed constraints, which stay closed, }
\end{aligned}
$$

and

$$
\lambda_{N_{i}}^{-} \approx \lim _{t \uparrow t_{i}} \lambda_{N}(t), \quad \lambda_{N_{i}}^{+} \approx \lim _{t \downarrow t_{i}} \lambda_{N}(t)
$$

Now, what is active for this half-explicit trapezoidal rule? We assume that the generalized position $q_{i-1}$ and the right-hand limit of the generalized velocity $v_{i-1}^{+}$are known at $t_{i-1}$ as illustrated in the graphical interpretation in Fig. 2. These values are set to the first stage of the generalized position (43) and of the generalized velocity (46), respectively. The generalized velocity is assumed to be continuous in the interior of the time interval. In the specific case of linear trial functions, there is a linear velocity propagation until its left-hand limit $v_{i-1,1}$ at $t_{i}$. Hence, the second stage (47) of the generalized velocity is calculated with a step of the explicit Euler method. Everything is known but for the right-hand limit of the contact force $\lambda_{N_{i-1}}^{+}$. We postpone the question whether to incorporate (49) for its calculation. From a general point of view subsequently, the second stage of the generalized position at $t_{i}$ is calculated with the trapezoidal rule (44). As the jump at $t_{i}$ occurs in such a way that the generalized velocity at $t_{i}$ is right-continuous, the left-hand limit of the generalized velocity $v_{i}^{-}$is corrected by a velocity jump leading to the right-hand limit of the generalized velocity $v_{i}^{+}$. The respective trapezoidal rule (48) involves the unknown left-hand limit of the contact force $\lambda_{N_{i}}^{-}$and the impulse $\Lambda_{N_{i}}$ as well as (50) and (31) if necessary. The effects of all impulses that would technically occur in the interior of the time interval are summarized at the end of the time interval.

The procedure of incorporating (49)-(50) and (31) can be implemented easily for a decoupled bouncing ball example (cf. Sect. 5.2 for gravitational acceleration in $-y$-direction) because there is only one single contact possibility [10, 27]. For multi-contact situations it is not clear how to decide which contacts shall be considered active 


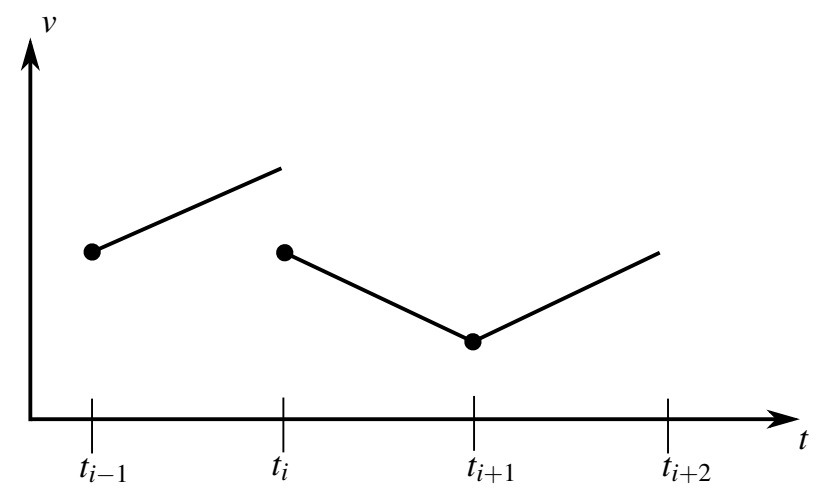

Figure 2: Interpretation of velocity jumps [37].

concerning the three different constraint equations (49)-(50) and (31). The half-explicit trapezoidal rule needs further interpretation for a consistent extension to simultaneous contact occurrences and nonlinear dynamics. Hence in comparison to [10], we develop a new technique to assign contact forces and impulses.

\subsection{Assignment of contact forces and impulses}

After having calculated the right-hand limit of the contact force $\lambda_{N_{i-1}}^{+}$at $t_{i-1}$ according to Sect. 2.4, we predict the left-hand limit of the generalized velocity at $t_{i}$, i.e., the second stage of the generalized velocity $v_{i-1,1}(47)$, and we calculate the second stage of the generalized position $q_{i-1,1}(44)$. This allows us to calculate the gap function $g_{N_{i}}$.

- If on the one hand there is no gap function which has been inactive $\left(g_{N_{k^{*}}}\left(q_{i-1,0}\right)>0\right)$ at $t_{i-1}$ and which now becomes active $\left(g_{N_{k^{*}}}\left(q_{i-1,1}\right) \leq 0\right)$ at $t_{i}$, no impact has to be considered. We set $\Lambda_{N_{i}}=0$ to fully profit of higher integration order and calculate the left-hand limit of the contact force $\lambda_{N_{i}}^{-}$at $t_{i}$ according to Sect. 2.4. Knowing $\lambda_{N_{i}}$, we improve the predicted left-hand limit of the generalized velocity at $t_{i}$ :

$$
v_{i}^{-}:=v_{i-1}^{+}+\frac{\Delta t_{i}}{2} M_{i-1}^{-1}\left[h_{i-1}^{+}+W_{N_{i-1}} \lambda_{N_{i-1}^{+}}^{+}\right]+\frac{\Delta t_{i}}{2} M_{i-1,1}^{-1}\left[h_{i-1,1}+W_{N_{i-1,1}} \lambda_{N_{i}}^{-}\right] .
$$

- If on the other hand there is at least one inactive gap function $\left(g_{N_{k^{*}}}\left(q_{i-1,0}\right)>0\right)$ at $t_{i-1}$ that now becomes active $\left(g_{N_{k^{*}}}\left(q_{i-1,1}\right) \leq 0\right)$, an impact occurs in the whole rigidly connected component of the impact source. Hence in this rigidly connected component, the calculation of $\lambda_{N_{i}}^{-}$is not consistent and does not improve the accuracy of the scheme. For the ease of description, we set $\lambda_{N_{i}}^{-}=0$ and also $\lambda_{N_{i-1}}^{+}=0$ everywhere in the multibody system and calculate the left-hand limit of the generalized velocity at $t_{i}$, i.e., the free velocity:

$$
v_{i}^{-}:=v_{i-1}^{+}+\frac{\Delta t_{i}}{2}\left\{M_{i-1}^{-1} h_{i-1}^{+}+M_{i-1,1}^{-1} h_{i-1,1}\right\} .
$$

This global effect does not occur in the decoupled bouncing ball example. For the computation of $v_{i}^{+}$and $\Lambda_{N_{i}}$, we write (6) on velocity level row-by-row:

$$
\Lambda_{N_{i}}= \begin{cases}0 & \text { if } g_{N}\left(q_{i-1,1}\right)>0 \\ \operatorname{proj}_{\mathbb{R}_{0}^{+}}\left[\Lambda_{N_{i}}-r \dot{\bar{g}}_{N_{i}}^{+}\right] & \text {else }\end{cases}
$$

with

$$
\dot{\bar{g}}_{N_{i}}^{+}=\dot{g}_{N_{i}}^{+}+\varepsilon_{N} \dot{g}_{N_{i}}^{-}
$$

Equation (5) can be used to eliminate $\dot{g}_{N_{i}}^{+}$, row-by-row resulting in

$$
\Lambda_{N_{i}, \mathscr{I}_{1}^{i-1,1}}=\operatorname{proj}_{\mathbb{R}_{0}^{+}}\left[\Lambda_{N_{i}, \mathscr{I}_{1}^{i-1,1}}-r \dot{\bar{g}}_{N_{i}, \mathscr{I}_{1}^{i-1,1}}^{+}\right]
$$


with $G_{N_{i}}:=G_{N}\left(q_{i}\right)$ and

$$
\dot{\bar{g}}_{N_{i}, \mathscr{I}_{1}^{i-1,1}}^{+}=G_{N_{i}, \mathscr{I}_{1}^{i-1,1}} \Lambda_{N_{i}, \mathscr{I}_{1}^{i-1,1}}+\left(I+\varepsilon_{N, \mathscr{I}_{1}^{i-1,1}}\right) W_{N_{i}, \mathscr{I}_{1}^{i-1,1}}^{T} v_{i}^{-}
$$

This equation is solved with a semi-smooth Gauss-Newton method.

We have to use structures from classic timestepping schemes and event-driven schemes to define the half-explicit trapezoidal rule.

\subsection{State increment}

Finally, we update the generalized velocity:

$$
v_{i}^{+}=v_{i}^{-}+M_{i}^{-1} W_{N_{i}} \Lambda_{N_{i}} .
$$

Hence in the impact-free case, it is $v_{i}^{+}=v_{i}^{-}$as expected. For the generalized position, it is

$$
q_{i}=q_{i-1}+\frac{\Delta t_{i}}{2}\left\{v_{i-1,0}+v_{i-1,1}\right\} \text {. }
$$

\subsection{Overview}

The overall algorithm can be summarized as shown in Fig. 3. More details can be found in the report [27].

\subsection{First analysis of simulation results}

Simulation results for the slider-crank mechanism in Fig. 1 of Sect. 2.1 with $\varepsilon_{N}=0.4$ and $\Delta t=10^{-4}$ s are depicted in Figs. 4 and 5. The curves show a nearly perfect behavior. The benefits from the time-discontinuous Galerkin schemes presented in [10] concerning theoretical investigations and a decoupled bouncing ball example can also be carried over to multi-contact examples. Impulsive and non-impulsive periods are separated; hence, higher-order trial functions can be used in non-impulsive periods whereas consistency is preserved for the impacts. Nevertheless, we improve the curves concerning the following observations. The penetration of the bordering wall occurs because timestepping schemes in general do not detect but only capture events, e.g. the time instant when a gap function closes. Contact activity means $g_{N_{i, l}} \leq 0 \wedge \dot{g}_{N_{i, l}}^{ \pm} \leq 0$ and not $g_{N_{i, l}}=0 \wedge \dot{g}_{N_{i, l}}^{ \pm}=0$. We will see that a velocity level discretization of the contact forces is a remedy for the drift-off effect and opens the path to include friction naturally. On acceleration level, the implementation of the friction transitions would also be rather complicated because of the larger combinatorial problem depth in comparison to a velocity level discretization. On velocity level, the nonlinearity in the equations of motion, e.g. $v_{\mathbf{m}}$, will be reduced. Nonlinearity in general induces the not always decreasing energy trend of the trapezoidal rule which differs from the midpoint rule in the nonlinear regime [14]. On acceleration level, contacts and impacts are defined by different activity rules using $\mathscr{I}_{2}$ and $\mathscr{I}_{1}$. As a compromise between acceleration and position level discretizations, the velocity level discretization will unify contact and impact activation rules.

\section{Half-explicit timestepping schemes on velocity level}

In this section, we reduce the drawbacks, i.e., the degree of nonlinearity and the drift-off effect, for half-explicit timestepping schemes on acceleration level from the preceding section by introducing a velocity level discretization:

$$
\begin{aligned}
q_{i-1,0} & =q_{i-1} \\
q_{i-1,1} & =q_{i-1}+\frac{\Delta t_{i}}{2}\left\{v_{i-1,0}+v_{i-1,1}\right\} \\
q_{i} & =q_{i-1}+\frac{\Delta t_{i}}{2}\left\{v_{i-1,0}+v_{i-1,1}\right\} \\
v_{i-1,0} & =v_{i-1}^{+} \\
v_{i-1,1} & =v_{i-1}^{+}+\Delta t_{i} M_{i-1}^{-1}\left[h_{i-1}^{+}+W_{N_{i-1}} \lambda_{N_{i-1}}^{+}+W_{T_{i-1}} \lambda_{T_{i-1}}^{+}\right] \\
v_{i}^{-} & =v_{i-1}^{+}+\frac{\Delta t_{i}}{2} M_{i-1}^{-1}\left[h_{i-1}^{+}+W_{N_{i-1}} \lambda_{N_{i-1}}^{+}+W_{T_{i-1}} \lambda_{T_{i-1}}^{+}\right]+\frac{\Delta t_{i}}{2} M_{i-1,1}^{-1}\left[h_{i-1,1}+W_{N_{i-1,1}} \lambda_{N_{i}}^{-}+W_{T_{i-1,1}} \lambda_{T_{i}}^{-}\right] \\
v_{i}^{+} & =v_{i}^{-}+M_{i}^{-1}\left[W_{N_{i}} \Lambda_{N_{i}}+W_{T_{i}} \Lambda_{T_{i}}\right]
\end{aligned}
$$




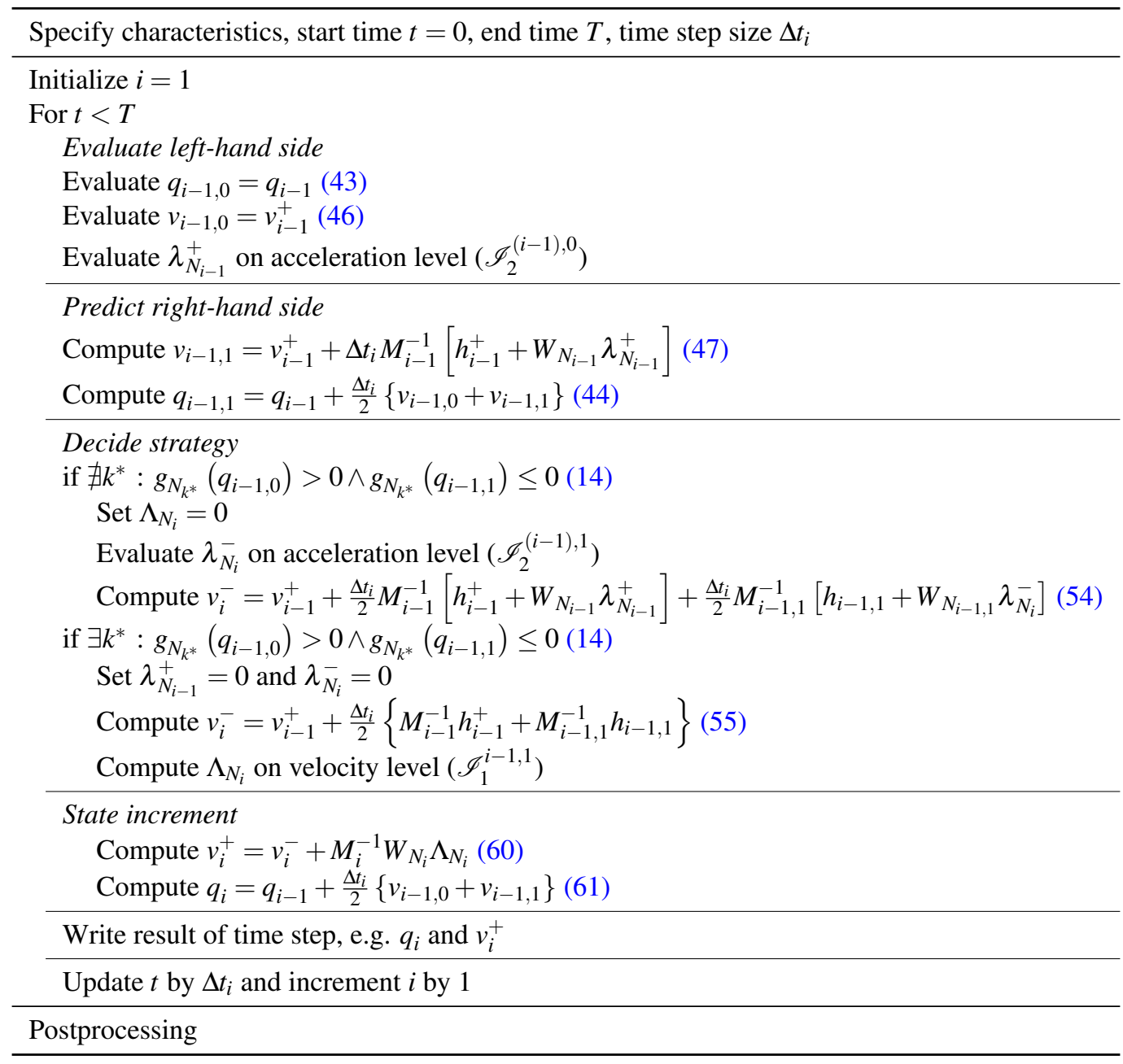

Figure 3: Flowchart of the half-explicit timestepping scheme on acceleration level.

using an implicit evaluation of constraints [2-8] for calculating both $\lambda_{N_{i-1}}^{+}, \lambda_{T_{i-1}}^{+}$and $\lambda_{N_{i}}^{-}, \lambda_{T_{i}}^{-}$. We solve the following equations for the second stage of the velocity

$$
\begin{aligned}
& 0=\lambda_{N_{i-1}, \mathscr{I}_{1}^{i-1,0}}^{+}-\operatorname{proj}_{\mathbb{R}_{0}^{+}}\left(\lambda_{N_{i-1}, \mathscr{I}_{1}^{i-1,0}}^{+}-r W_{N, \mathscr{I}_{1}^{i-1,0}}^{T}\left(q_{i-1}+\Delta t_{i} v_{i-1}^{+}\right) v_{i-1,1}\right), \\
& 0=\lambda_{T_{i-1}, \mathscr{I}_{1}^{i-1,0}}^{+}-\operatorname{proj}_{C_{T}\left(\lambda_{N_{i-1}, \mathscr{I}_{1}^{i-1,0}}^{+}\right.}\left(\lambda_{T_{i-1}, \mathscr{I}_{1}^{i-1,0}}^{+}-r W_{T, \mathscr{I}_{1}^{i-1,0}}^{T}\left(q_{i-1}+\Delta t_{i} v_{i-1}^{+}\right) v_{i-1,1}\right)
\end{aligned}
$$

and the following equations assuming that the output equation of the trapezoidal rule defines a virtual third stage:

$$
\begin{aligned}
& 0=\lambda_{N_{i}, \mathscr{I}_{1}^{i-1,1}}^{-}-\operatorname{proj}_{\mathbb{R}_{0}^{+}}\left(\lambda_{N_{i}, \mathscr{I}_{1}^{i-1,1}}^{-}-r W_{N_{i}, \mathscr{I}_{1}^{i-1,1}}^{T} v_{i}^{-}\right) \\
& 0=\lambda_{T_{i}, \mathscr{I}_{1}^{i-1,1}}^{-}-\operatorname{proj}_{C_{T}\left(\lambda_{N_{i}, \mathscr{I}_{1}^{i-1,1}}^{-}\right)}\left(\lambda_{T_{i}, \mathscr{I}_{1}^{i-1,1}}^{-}-r W_{T_{i}, \mathscr{I}_{1}^{i-1,1}}^{T} v_{i}^{-}\right) .
\end{aligned}
$$

It is important to evaluate $W$ on the right-hand side of the interval. As we do not know its value for the second stage of the velocity, we have to calculate a prediction, which, however, is not used for the calculation of any index set. The virtual third stage of the velocity coincides with the output equation of the trapezoidal rule. Hence, the velocity 


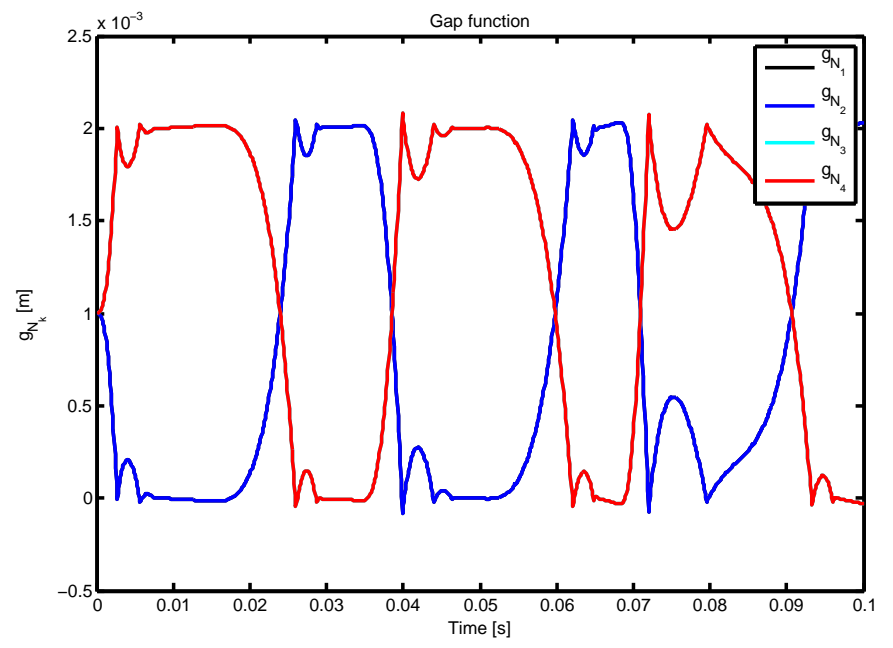

Figure 4: Normal gap functions of the slider for half-explicit timestepping on acceleration level.

level constraint is automatically satisfied also for the beginning of the next time step. We have easily included friction which naturally is stated on velocity level with

$$
\lambda_{T_{i}}^{-} \approx \lim _{t \uparrow_{i}} \lambda_{T}(t), \quad \lambda_{T_{i}}^{+} \approx \lim _{t \downarrow t_{i}} \lambda_{T}(t) .
$$

The active normal impact equations on velocity level are given by (56). The active tangential impact equations on velocity level are similar to (32). We use a fixed parameter $r$. The overall algorithm can be summarized as shown in Fig. 6. An intermediate velocity level discretization can be found in the report [27].

With the half-explicit evaluation of the constraints, we get the typical results of a velocity level discretization in comparison to the acceleration level discretization (Fig. 7) discussing the slider-crank mechanism in Fig. 1 of Sect. 2.1 with $\varepsilon_{N}=0.4$ and $\Delta t=10^{-4} \mathrm{~s}$.

\section{Multi-contact examples}

In this section, we discuss several examples to show the benefits of the proposed scheme. The slider-crank mechanism is a nonlinear example and the bouncing ball in a box is a linear example. We compare computational results, convergence and computing time of the half-explicit timestepping schemes on acceleration and velocity level, as well as of the classic explicit Moreau-Jean timestepping scheme. The computations have been done with Matlab. Thereby index sets are calculated without any additional tolerances. The constraints are calculated with a tolerance of $10^{-12} \mathrm{~m}$ for satisfying the constraint equations.

\subsection{Slider-crank mechanism}

For the slider-crank mechanism in Sect. 2.1, we give some supplementary impressions without regarding friction. The calculations have been performed with the half-explicit timestepping scheme on velocity level. Figure 8 demonstrates nicely the splitting of the different Lagrange multipliers and therefore the possibility to achieve locally a higher order discretization. Further insight is left to the report [27]. 


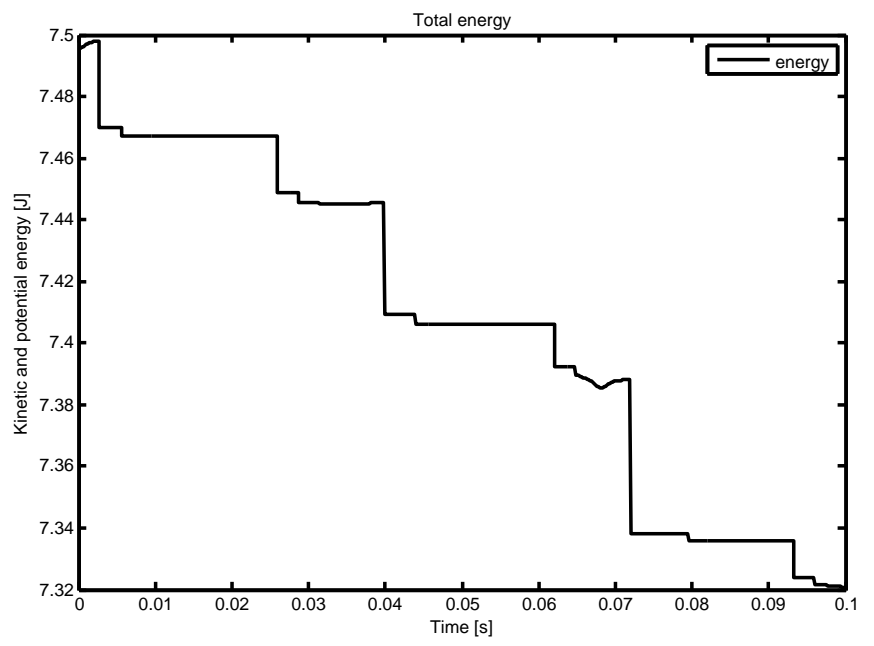

Figure 5: Total energy of the slider-crank mechanism for half-explicit timestepping on acceleration level.

\subsubsection{Convergence and computing time for the bilateral case}

Regarding a bilateral slider-crank mechanism without friction, i.e., $c=0 \mathrm{~m}$, we compare the half-explicit timestepping scheme on velocity level with the classic explicit Moreau-Jean timestepping scheme. Thereby, we analyze computing time and convergence of the schemes in comparison with a corresponding Simpack model, which is integrated with the event-driven SODASRT2 solver and a high tolerance $10^{-12}$.

Independent of different time step-sizes, the relative overhead of the half-explicit timestepping scheme on velocity level in comparison with the classic explicit Moreau-Jean timestepping scheme is about 1.2. Hence, the computation time per time step is about 1.2 times larger for the half-explicit timestepping scheme on velocity level. We calculate the error with respect to the reference Simpack solution for different time step-sizes. Thereby, we compute the differences of the generalized coordinates for the considered time instances and arrange them in a matrix. The 2-norm of this matrix results in Table 2. Concerning these evaluations, this is a trend to an order of convergence of 1 for the classic

\begin{tabular}{lccc}
\hline$\Delta t[\mathrm{~s}]$ & $10^{-3}$ & $10^{-4}$ & $10^{-5}$ \\
\hline error of the classic explicit Moreau-Jean timestepping scheme & $6.9 \cdot 10^{-1}$ & $2.1 \cdot 10^{-1}$ & $6.6 \cdot 10^{-2}$ \\
error of the half-explicit timestepping scheme on velocity level & $4.2 \cdot 10^{-1}$ & $2.4 \cdot 10^{-2}$ & $8.4 \cdot 10^{-4}$ \\
\hline
\end{tabular}

Table 2: Bilateral slider-crank example: comparison of the error for different time step-sizes.

explicit Moreau-Jean timestepping scheme and to an order of convergence of 2 for the half-explicit timestepping scheme on velocity level. The overall relative cost to achieve a given tolerance, e.g. $10^{-2}$, is given by the relation of the fractions of the relative overhead and the necessary time step-size, i.e., $1.2 \cdot 10^{-1}$ for the half-explicit timestepping scheme on velocity level in comparison to the classic explicit Moreau-Jean timestepping scheme. Thus, it makes sense to apply higher-order approximations for appropriate examples.

\subsubsection{Convergence and computing time for the unilateral case}

Regarding a unilateral slider-crank mechanism with friction, we compare the half-explicit timestepping scheme on velocity level with the classic explicit Moreau-Jean timestepping. Thereby, we analyze computing time and convergence of the schemes assuming a reference solution given by a simulation of the half-explicit timestepping on velocity level with $\Delta t=10^{-7} \mathrm{~s}$. 


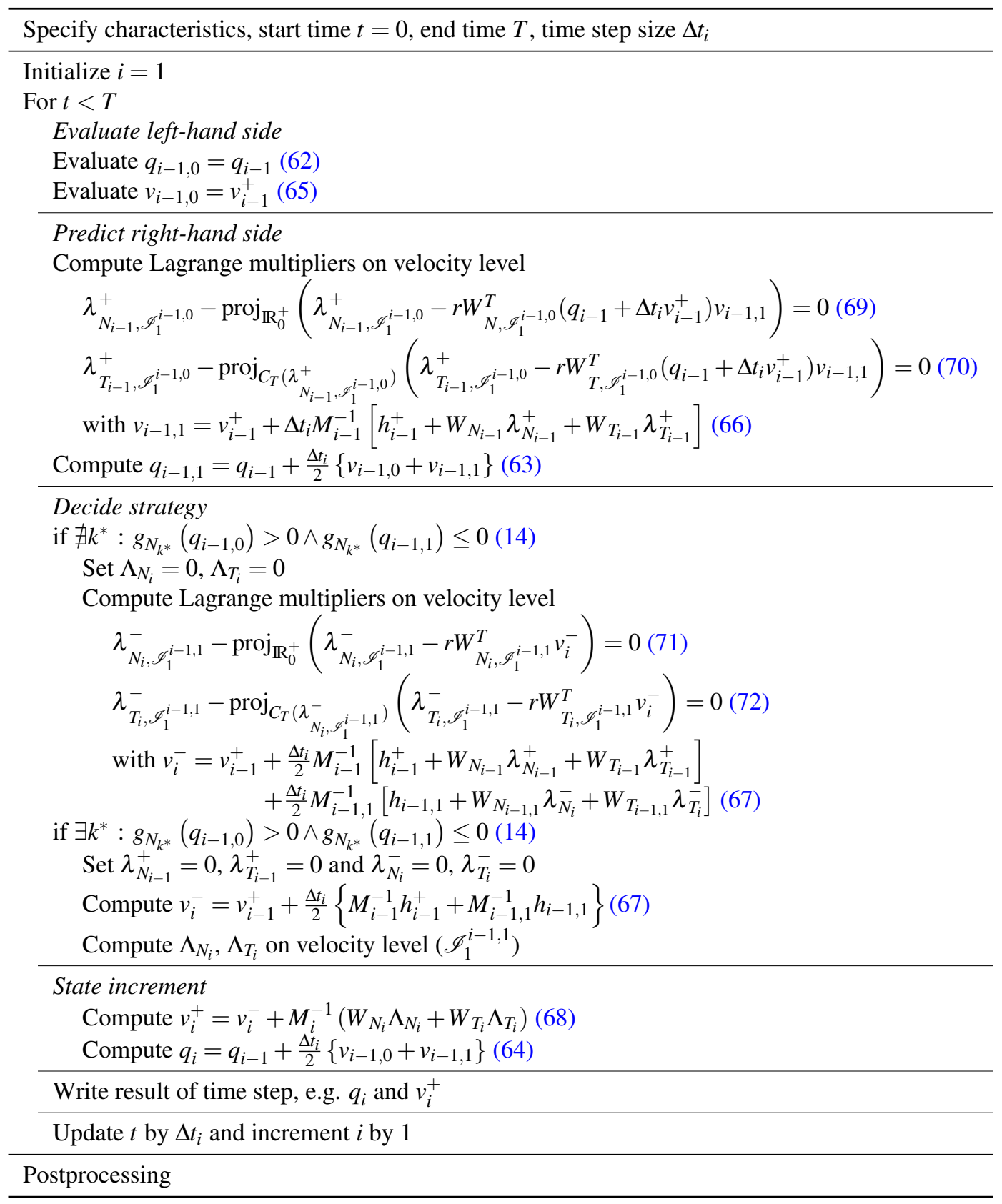

Figure 6: Flowchart of the half-explicit timestepping scheme on velocity level.

The relative overhead of the half-explicit timestepping scheme on velocity level is about 1.15 . For the error, we get Table 3. The order of convergence concerning these evaluations drops down to 1 for both integration schemes. However, the results for the half-explicit timestepping scheme on velocity level are better perhaps because of the possibility to achieve a locally higher order. The overall relative cost for a given tolerance, e.g. $10^{-2}$, is $1.15 \cdot 10^{-1}$ for the half-explicit timestepping scheme on velocity level in comparison to the classic explicit Moreau-Jean timestepping scheme. Hence like in the bilateral case, the relative overhead could be compensated by an adaptive time-step size and a control of the accuracy. 


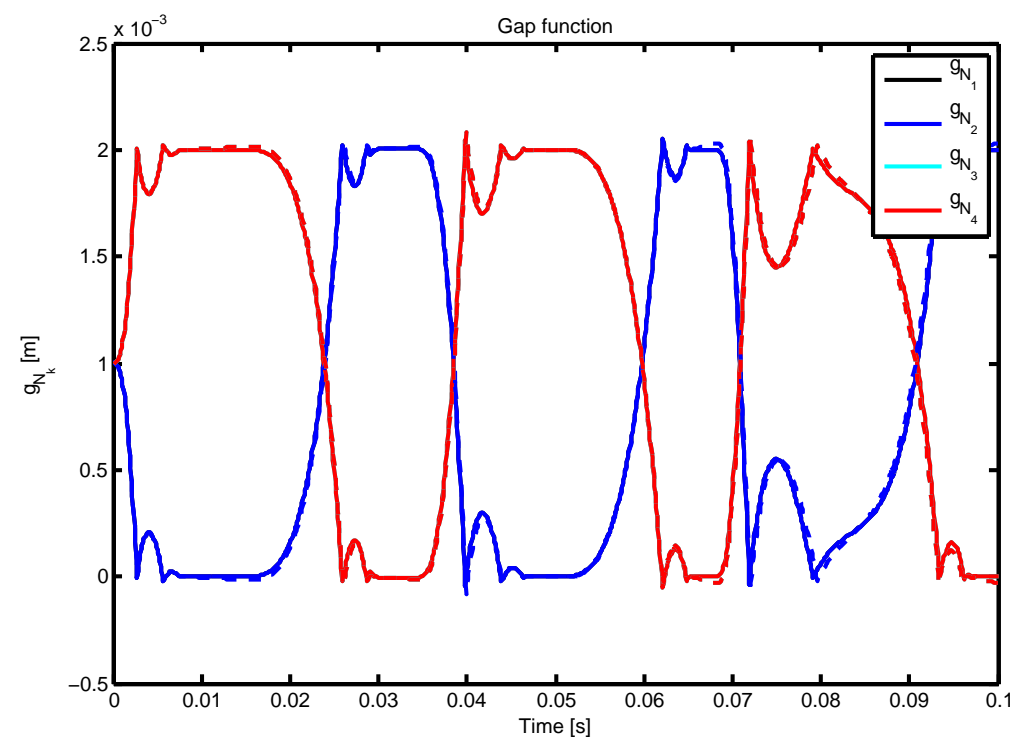

Figure 7: Normal gap functions of the slider for half-explicit timestepping on acceleration (dashed) and velocity level (solid).

\begin{tabular}{lccc}
\hline$\Delta t[\mathrm{~s}]$ & $10^{-3}$ & $10^{-4}$ & $10^{-5}$ \\
\hline error of the classic explicit Moreau-Jean timestepping scheme & $7.1 \cdot 10^{-1}$ & $1.5 \cdot 10^{-1}$ & $4.5 \cdot 10^{-2}$ \\
error of the half-explicit timestepping scheme on velocity level & $4.7 \cdot 10^{-1}$ & $1.8 \cdot 10^{-2}$ & $3.3 \cdot 10^{-3}$ \\
\hline
\end{tabular}

Table 3: Unilateral slider-crank example: comparison of the error for different time step-sizes.

\subsection{Bouncing ball in a box}

The linear bouncing ball in a box example discusses the drift-off effect and explains the energy behavior of halfexplicit timestepping schemes based on the trapezoidal rule.

A bouncing ball of radius $R$ in a planar box of width $a$ and height $b$ (Fig. 9) is described by the two translational coordinates, $x$ and $y$, of its center of gravity. The rigid ball with mass $m$ is subject to gravitation $\gamma$ in $-(\cos (\pi / 6) \sin (\pi / 6))^{T}$-direction. The normal gap functions $g_{N}$ are shown in Fig. 9. The vector containing the coefficients of restitution is $\varepsilon_{N}$. Friction is not considered. Fixed characteristics are given in Table 4; further information is left to the report [27].

For the application of the half-explicit timestepping methods based on the trapezoidal rule to the slider-crank mechanism, we have mentioned a not always decreasing energy trend (Fig. 5). As we see in Fig. 10, the energy behavior for the linear bouncing ball in a box example actually shows the expected curve without any increasing periods. The previous non-decreasing trend is traced back to the nonlinear nature of the example. In Fig. 11, we see the normal gap functions. They coincide for both discretization schemes because of the linearity of the example. All calculations have been performed with $\Delta t=5 \cdot 10^{-3} \mathrm{~s}$.

\section{Flexible examples}

The elastic bar is a linear but flexible example and the rotor is a linear and flexible example from practice. In this section, we discuss the behavior of waves and damping for half-explicit timestepping schemes on velocity level. 

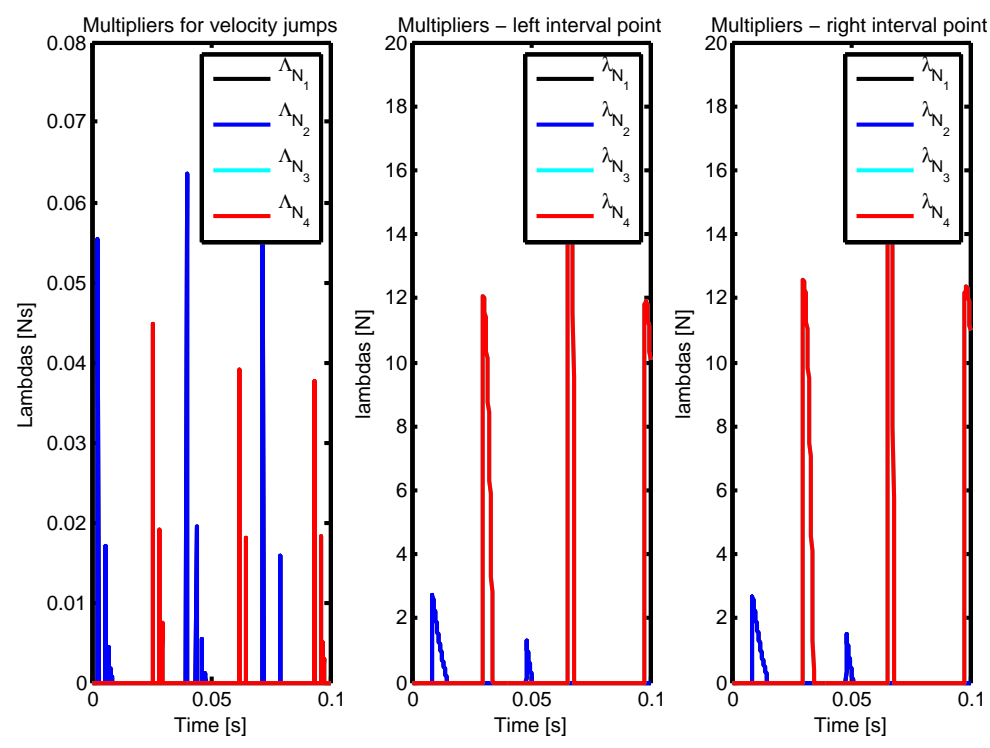

Figure 8: Lagrange multipliers of the slider-crank mechanism for half-explicit timestepping on velocity level.

\begin{tabular}{ll}
\hline Geometrical characteristics & $a=4.0 \mathrm{~m}$ \\
& $b=4.0 \mathrm{~m}$ \\
& $R=1.0 \mathrm{~m}$ \\
\hline Inertia properties & $m=1.0 \mathrm{~kg}$ \\
\hline Force elements & $\gamma=9.81 \mathrm{~m} / \mathrm{s}^{2}$ in negative $y$-direction \\
\hline Contact parameters & $\varepsilon_{N_{1}}=\varepsilon_{N_{2}}=\varepsilon_{N_{3}}=\varepsilon_{N_{4}}=0.3$ \\
\hline Initial conditions & $x_{0}=2.0 \mathrm{~m}$ \\
& $y_{0}=2.0 \mathrm{~m}$ \\
& $\dot{x}_{0}=0.0 \mathrm{~m} / \mathrm{s}$ \\
& $\dot{y}_{0}=0.0 \mathrm{~m} / \mathrm{s}$ \\
\hline
\end{tabular}

Table 4: Characteristics of the bouncing ball example.

\subsection{Elastic bar}

In this section, we consider the classical example of a linear elastic bar that impacts a rigid obstacle at constant velocity $v_{0}$. The example is depicted in Figure 12 . The bar has a length $L$, a section area $S$, a density $\rho$ and a Young modulus $E$. If we denote by $c_{0}=\sqrt{E / \rho}$ the longitudinal wave speed, the time of contact is twice the time of the traveling of elastic waves in the bar, that is $T=2 L / c_{0}$. Within the contact time, the contact force is constant and equals $\tau=E S v_{0} / c_{0}$. The parameters for this example are summarized in Table 5. Some further information can be found in the report [27]. The bar is discretized by $N$ linear rod finite elements. The first simulation results with the half-explicit timestepping scheme on velocity level for a time-step size $\Delta t=10^{-7} \mathrm{~s}$ and 50 finite elements are reported in Fig. 13. This simulation has been carried out with Siconos, an open-source software for the modelling and the simulation of nonsmooth dynamical systems [60]. In this simulation, the expected solution for the elastic bar is found exactly with Lemke's algorithm. The time of contact of the bar is well-approximated and the velocity within the contact interval is maintained at zero. The violation of the constraint is equal to $5 \cdot 10^{-9} \mathrm{~m}$. The presence of the impulse is a consequence of the space discretization by finite elements. The finite mass of the node in contact is instantaneously stopped at the impact. This generates an impulse. This impulse associated with a plastic impact induces a loss of the total energy at impact which can be observed in the energy plot. Finally, we remark that the reaction force is well approximated in 


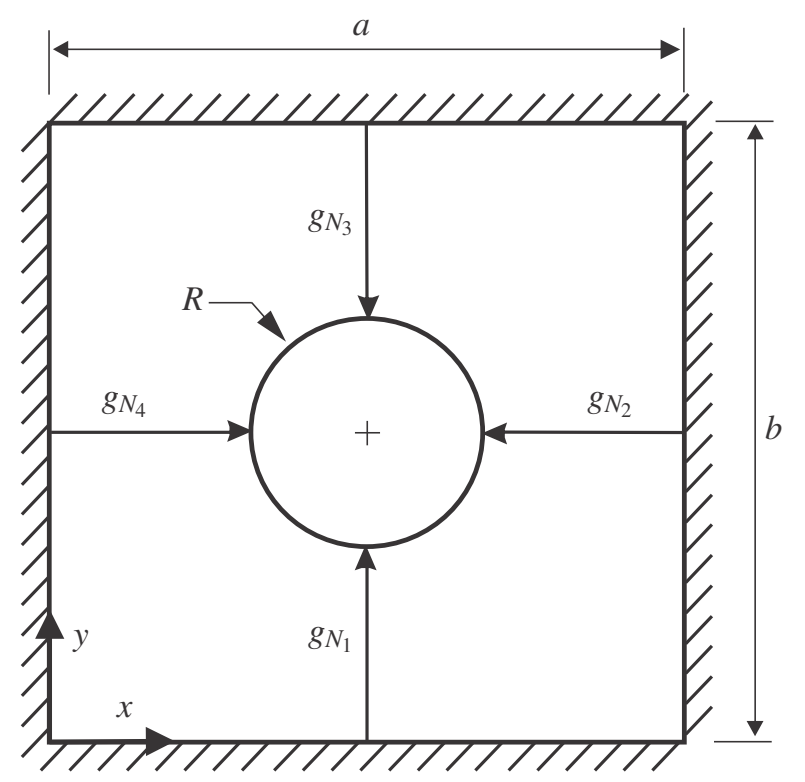

Figure 9: Bouncing ball in a box.

\begin{tabular}{ll}
\hline Geometrical characteristics & $L=1 \mathrm{~m}$ \\
& $S=\pi \cdot 10^{-4} \mathrm{~m}^{2}$ \\
\hline Material properties & $\rho=7800 \mathrm{~kg} / \mathrm{m}^{3}$ \\
& $E=2.1 \cdot 10^{1} \mathrm{~N} / \mathrm{m}^{2}$ \\
\hline Initial conditions & $v_{0}=0.1 \mathrm{~m} / \mathrm{s}$ \\
\hline Solution characteristics & $c_{0}=5.188 \cdot 10^{3} \mathrm{~m} / \mathrm{s}$ \\
& $T=3.854 \cdot 10^{-4} \mathrm{~s}$ \\
& $\tau=1.271 \cdot 10^{3} \mathrm{~N}$ \\
\hline
\end{tabular}

Table 5: Characteristics of the elastic bar example.

the first half of the contact period before the development of an instability which is inherent to standard finite element approximations of a travelling velocity jump trough the mesh. Nevertheless, it is noteworthy that the contact is never lost due to artificial numerical chattering.

In Figure 14, a similar simulation is reported but the number of elements is increased to 1000 . We observe that the reaction impulse is reduced due to the decreased mass of the node in contact. The energy loss is also reduced. The instability is nevertheless still visible but its frequency has increased with the inverse of the element size. The travelling of the impulse excites the spurious high frequency mode associated with the discretization. One of the usual ways to circumvent this problem is to introduce an artificial damping in the simulation. The discretization family presented in this paper is explicit concerning the internal forces and does not include numerical damping - even if it seems possible, it is left as further work [50]. Hence, the stability domain is bounded [7, 8]. That is why, we add a small amount of Rayleigh damping (proportional to the stiffness) to damp out the numerical artifact. Equivalently, we could add physical or material damping to the structure. In Figure 15, the results with the viscous damping are plotted. The stiffness proportional damping coefficient is equal to $\delta=10^{-3} / E \mathrm{~s}=4.761 \cdot 10^{-6} \mathrm{~s}$. We observe that the instability is nearly damped out and the post velocity is smooth. Only a remaining impulse travels trough the bar. The decay of the total energy can be seen in the energy plot.

If we compare our results with those in [44] for the standard Moreau-Jean timestepping scheme for the example of the elastic bar, we observe the same type of instability of the reaction forces when there is no numerical damping in the Moreau-Jean algorithm. Nevertheless, the proposed scheme is mainly interesting for two reasons. First, it enables a splitting between the reaction force and the artificial reaction impulse due to the space discretization. The value 


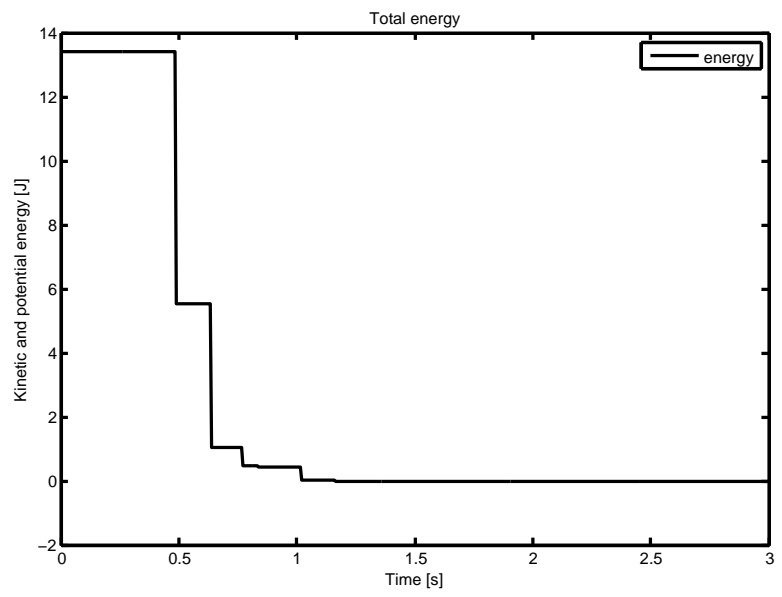

Figure 10: Total energy of the bouncing ball in a box for half-explicit timestepping on velocity level.

of the reaction force and the stress in the bar can be used for design as a good approximation of the forces in the space continuous bar. The magnitude of the reaction impulse gives a measure of the quality of the mesh with respect to the contact representation. Second, the scheme is half-explicit and thus requires less computational effort for the evaluation of the constitutive behavior in the nonlinear setting. However, we have to notice that a CFL type condition has to be satisfied since the internal forces are explicitly evaluated.

\subsection{Rotor}

A rotor test rig at the Institute of Applied Mechanics of the Technische Universität München (Fig. 16) is modelled in the multibody simulation framework MBSim [61, 62]. The idea of this example is the implementation of a variant of the half-explicit timestepping scheme on velocity level in MBSim and the application to an industrial example. Thereby, we test a simple and heuristic adaptation of the time-step size.

The rotor in Fig. 16 consists of a drive system with a driving torque about the rotor's longitudinal axis $T_{D}$ and a failure given by a torque about the vertical axis $T_{F}$. The drive system is mounted by a spring-damper element with constants $c_{D}, d_{D}$. The rotor's axle is modelled with $N_{A}$ beam elements in a floating frame of reference framework. Each finite element node has five degrees of freedom, two small out-off plane translational deflections, two small out-off plane rotational deflections and the rotation about the rotor's longitudinal axis. The length of the axle is $l_{A}$, its radius is $r_{A}$, the density is $\rho_{A}$, the Young's modulus is $E_{A}$, and the shear modulus is $G_{A}$. A mass proportional damping $d_{1_{A}}$ and a torsional damping $d_{2_{A}}$ are used. The fly-wheel is a rigid body with originally six degrees of freedom, which is mounted bilaterally at position $x_{F}$ from the axle's left end on the rotor's axle. It has the mass $m_{F}$ and the rotational inertia about the rotor's longitudinal axis $J_{F}$. The journal bearing consists of a rigid frustum with six degrees of freedom mounted bilaterally at its center at the left end of the rotor's axle but with negligible inertia values. This frustum of radius $r_{J F}$ and half width $b_{J F}$ moves freely in a circular bearing with radius $r_{J B}$, i.e., backlash and dry friction $\mu$. The bearing is a rigid body with six degrees of freedom, mass $m_{J B}$ and negligible rotational inertia values. The bearing is mounted at its center by a spring-damper element with constants $c_{J B}, d_{J B}$. The actual contact may only occur at position $x_{J B}$ from the axle's left end. We consider the bearing, e.g., as an auxiliary bearing occurring in many rotor systems with active magnetic bearings [63] or as an example for a safety bearing. The whole system is subject to gravitation $\gamma$ in negative vertical direction. The characteristics are summarized in Table 6. Damping is added because of the experience in Sect. 6.1.

For an implementation of a variant of the half-explicit timestepping scheme on velocity level in MBSim, we have to discretize

$$
\dot{q}=Y(q) v
$$




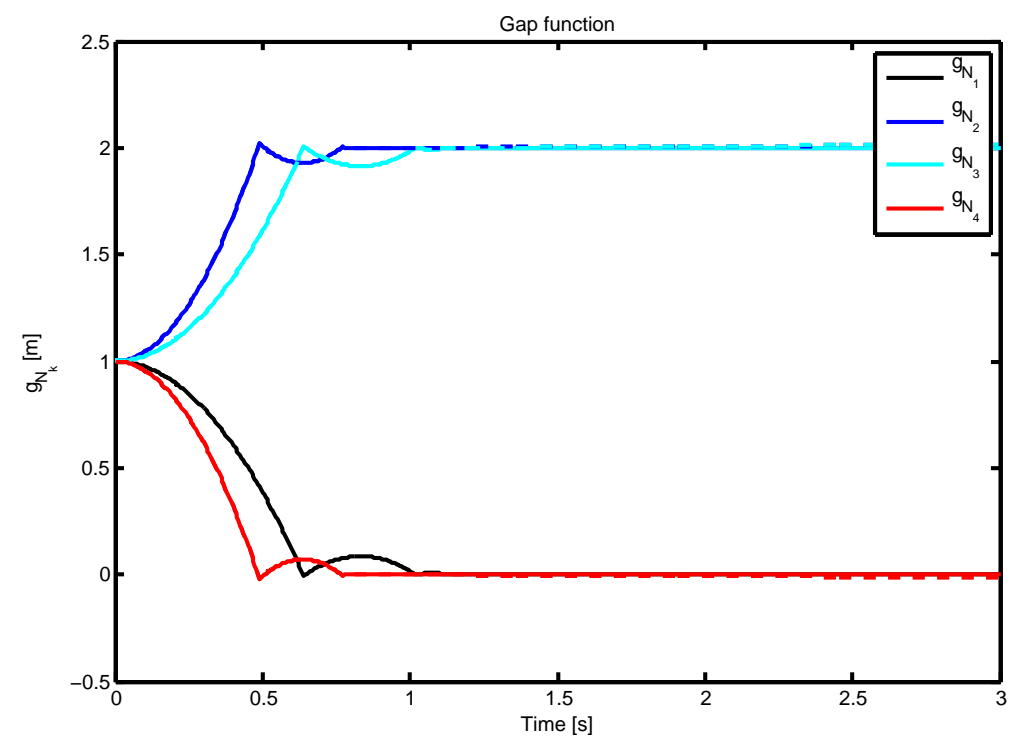

Figure 11: Normal gap functions of the bouncing ball in a box for half-explicit timestepping on acceleration (dashed) and velocity level (solid).

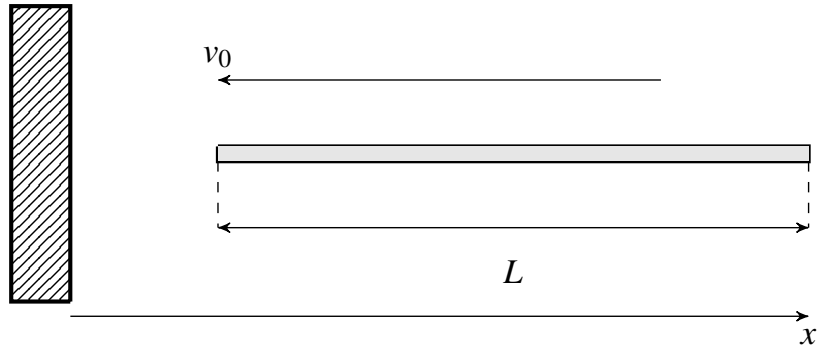

Figure 12: Linear elastic impacting bar on a rigid obstacle.

instead of $\dot{q}=v$. The matrix $Y$ is the transformation matrix between generalized velocities and the time-derivative of generalized coordinates; it is often used for the description of spatial rotations in multibody dynamics. At the same time, we adapt the interpretation of the contact activity for the first stage unknowns. Instead of (69)-(70), we solve

$$
\begin{aligned}
& 0=\lambda_{N_{i-1}, \mathscr{I}_{1}^{i-1, *}}^{+}-\operatorname{proj}_{\mathbb{R}_{0}^{+}}\left(\lambda_{N_{i-1}, \mathscr{I}_{1}^{i-1, *}}^{+}-r W_{N, \mathscr{I}_{1}^{i-1, *}}^{T}\left(q_{i-1}+\Delta t_{i} v_{i-1}^{+}\right) v_{i-1,1}\right), \\
& 0=\lambda_{T_{i-1}, \mathscr{I}_{1}^{i-1, *}}^{+}-\operatorname{proj}_{C_{T}\left(\lambda_{N_{i-1}, \mathscr{I}_{1}^{i-1, *}}\right)}\left(\lambda_{T_{i-1}, \mathscr{I}_{1}^{i-1, *}}^{+}-r W_{T, \mathscr{I}_{1}^{i-1, *}}^{T}\left(q_{i-1}+\Delta t_{i} v_{i-1}^{+}\right) v_{i-1,1}\right)
\end{aligned}
$$

with

$$
\mathscr{I}_{1}^{i-1, *}=\left\{k \in \mathscr{I}_{0}: g_{N_{k}}\left(q_{i-1}+\Delta t_{i} v_{i-1}^{+}\right) \leq 0\right\}
$$

in a more natural way. The time-step size for impulsive periods $\tilde{\Delta t}$ is heuristically adapted concerning the experiences with the slider-crank example (Table 3): $\tilde{\Delta t}=\frac{\Delta t}{10}$. As we do not know the error constant, we prefer this heuristic in comparison to $\tilde{\Delta t}=\Delta t^{2}$, which uses the non-impulsive order of the schemes. The overview and an impression of the forward whirl phenomenon are left to the report [27]. 


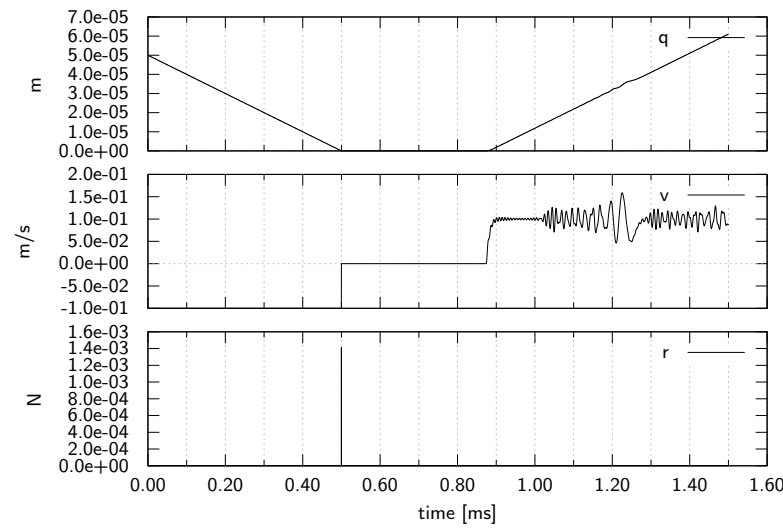

(a) position, velocity and reaction at the contact point

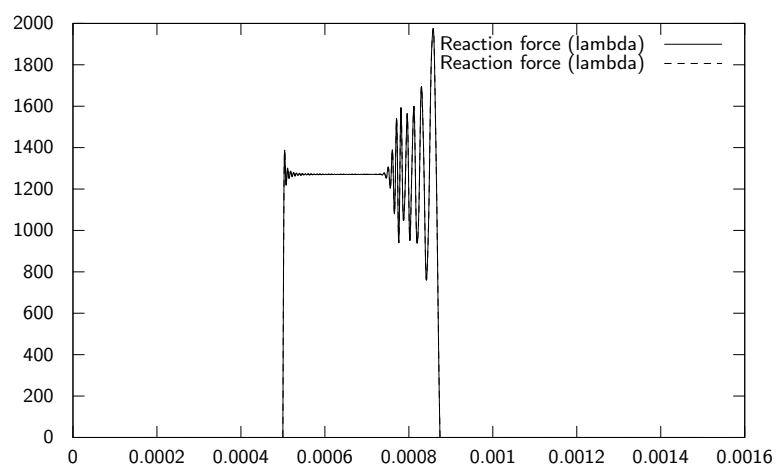

(c) reaction forces $\lambda$

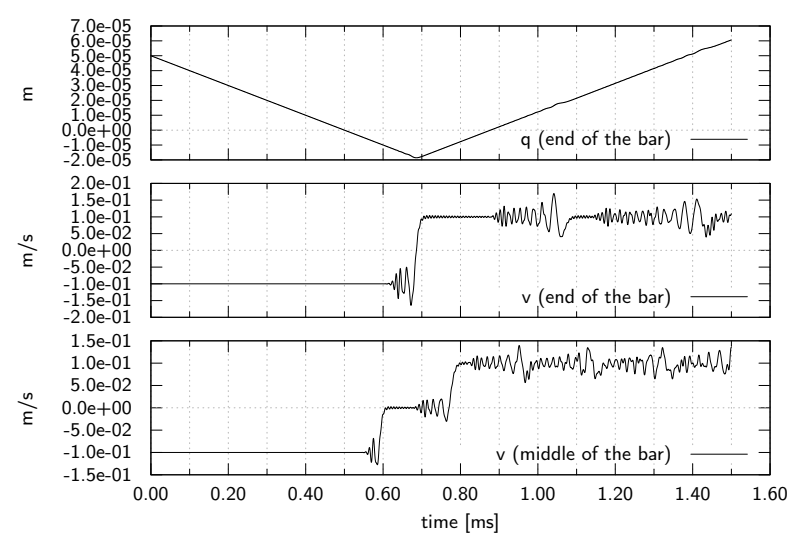

(b) position, velocity at the tip and velocity in the middle

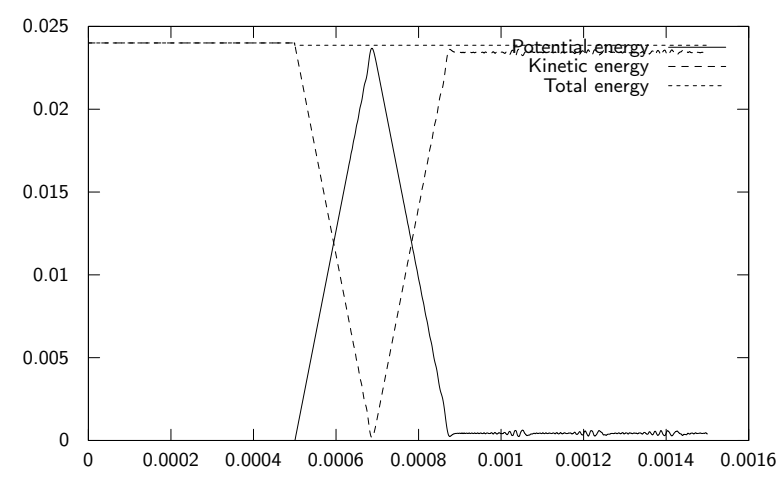

(d) kinetic, potential and total energy

Figure 13: Elastic bar simulation with the half-explicit timestepping scheme on velocity level $\Delta t=1 \cdot 10^{-7} \mathrm{~s}, N=50$.

We are interested in the general behavior of the variant of the half-explicit timestepping scheme on velocity level. In MBSim, the tolerances define tubes around the corner laws [64]. Starting from the impulsive tolerances $t^{\prime} l_{\Lambda}=10^{-10} \mathrm{Ns}, \operatorname{tol}_{\dot{g}}=10^{-10} \mathrm{~m} / \mathrm{s}$, we use $t o l_{\lambda}=\frac{t o l_{\Lambda}}{\Delta t}, t_{o l} l_{\ddot{g}}=\frac{t o l_{\dot{g}}}{\Delta t}$ for the calculation of the constraint equations with a single-step fixed-point method [64]. The index sets are detected with a tolerance of $10^{-8} \mathrm{~m}$. The variant of the half-explicit timestepping scheme on velocity level uses a fraction of impulsive integration steps of $1.3 \%$. The overall computing time of the variant of the half-explicit timestepping scheme on velocity level in comparison with the half-explicit variant of the Moreau-Jean timestepping scheme in [61] is 0.32. The total time of the analysis is about $850 \mathrm{~s}$ for the Moreau-Jean timestepping scheme. Hence, the new proposed scheme offers huge computing time saving potentials. In the calculations, we have used $\Delta t=5 \cdot 10^{-6} \mathrm{~s}$.

\section{Summary and Conclusion}

We have presented a time-discretization scheme for the simulation of nonsmooth mechanical systems with friction and impacts as well as rigid and flexible bodies with all possible interactions. Without switching between impulsive and non-impulsive periods, the time-discretization scheme is both consistent and efficient. It represents impulses, if necessary, and automatic local order elevation for state variables, if possible, at the same time. Constraints are formulated on velocity level in an implicit way using an augmented Lagrangian technique with semi-smooth Newton schemes without penetration; all other evaluations are explicit, which yields a half-explicit method. The scheme is derived and explained in detail concerning different criteria and it is applied to various examples with multi-contact, flexibility and industrial relevance. As a compromise between classic timestepping schemes and event-driven integration in the context of time-discontinuous Galerkin methods finally, two half-explicit timestepping schemes on velocity 


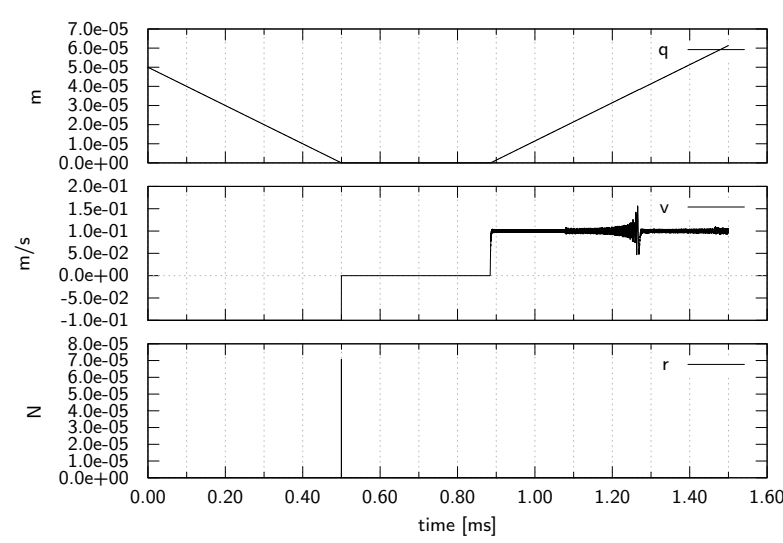

(a) position, velocity and reaction impulse at the contact point

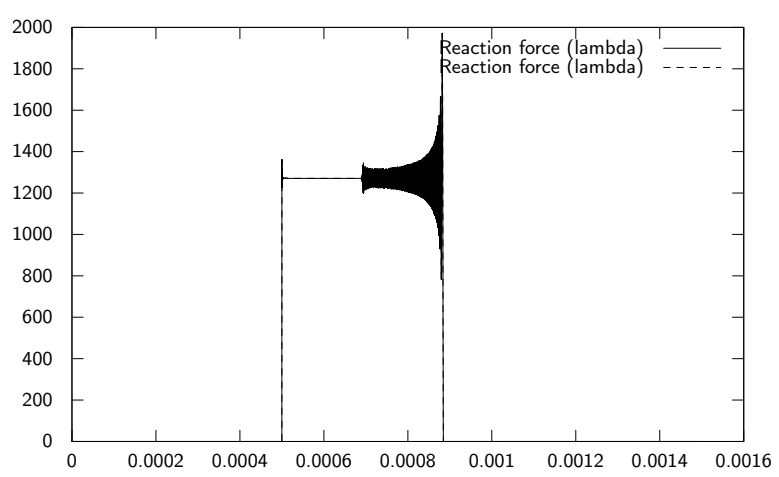

(c) reaction forces $\lambda$

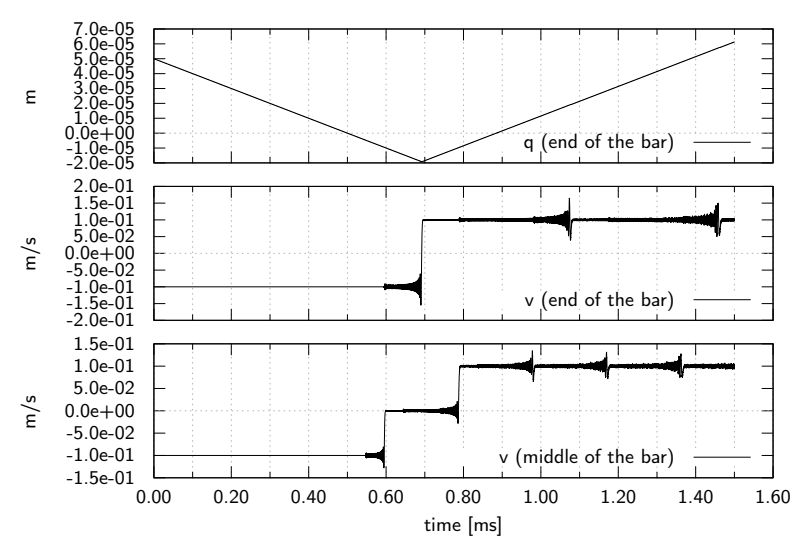

(b) position, velocity at the tip and velocity in the middle

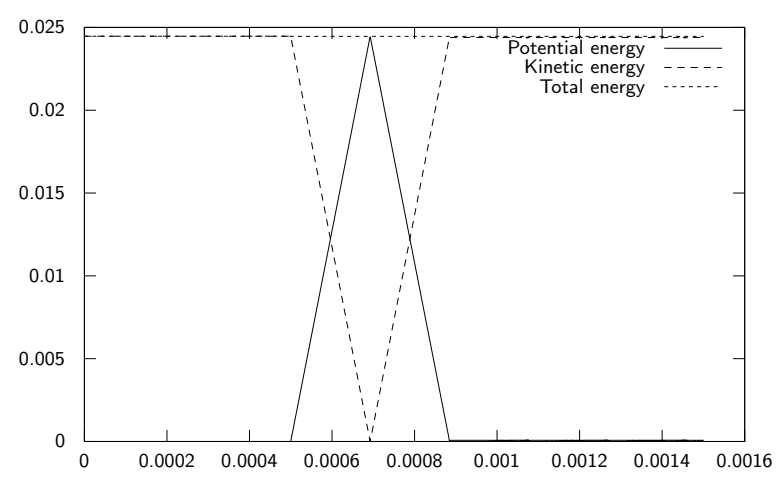

(d) kinetic, potential and total energy

Figure 14: Elastic bar simulation with the half-explicit timestepping scheme on velocity level $\Delta t=1 \cdot 10^{-7} \mathrm{~s}, N=1000$.

level distinguish impulsive and non-impulsive periods for all examples in a geometrically consistent way and reduce the computing time significantly. For flexible examples, we recognize the drawback of explicit schemes, i.e., stability issues, which can be solved by applying schemes from the Newmark family as base integration schemes in the framework of foreseen velocity jumps and respective impulses at the end of each discretization interval. This is left for future work.

\section{Appendix A. Half-explicit timestepping schemes on velocity level in the sense of Brasey [2]}

For the half-explicit trapezoidal rule, we adapt the interpretation of how to evaluate the constraints. Hence, we keep the interpretation as a time-discontinuous Galerkin method. However, we can also interpret the scheme with the 


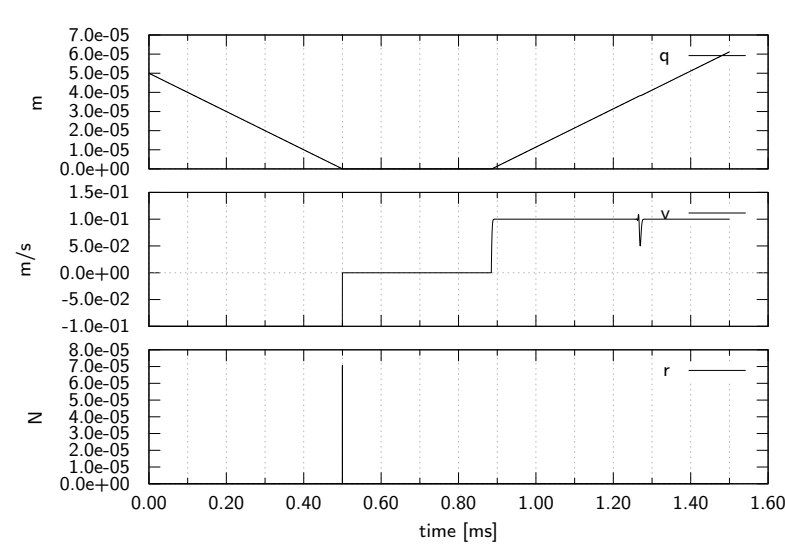

(a) position, velocity and reaction impulse at the contact point

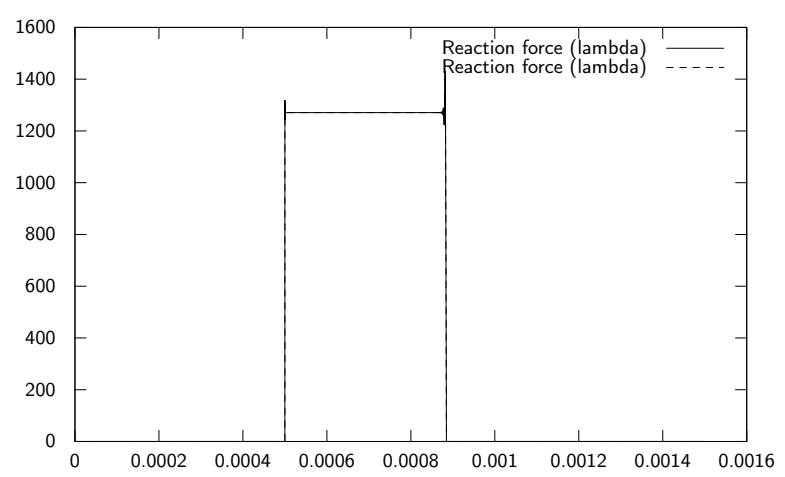

(c) reaction forces $\lambda$

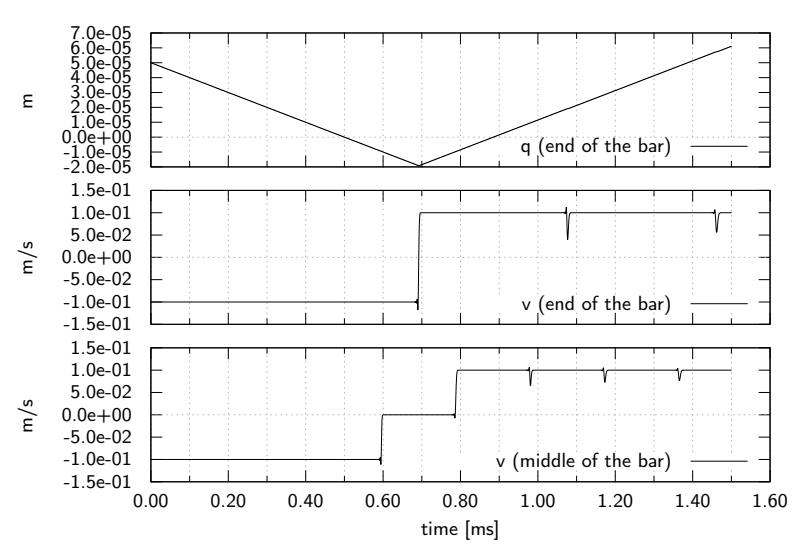

(b) position, velocity at the tip and velocity in the middle

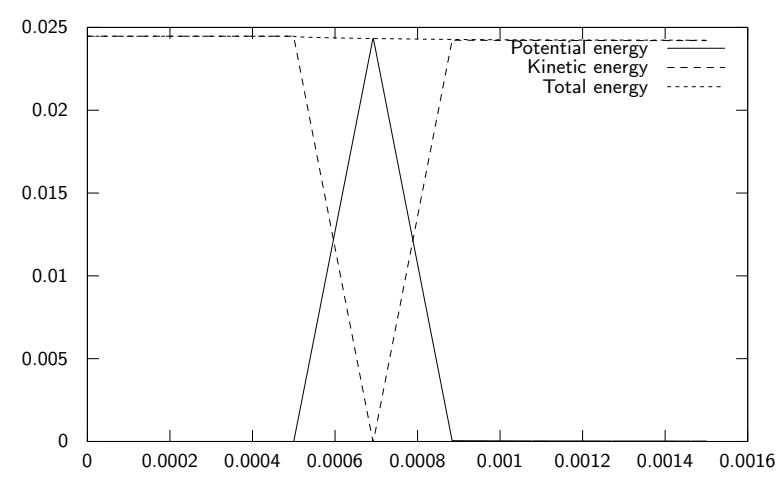

(d) kinetic, potential and total energy

Figure 15: Damped elastic bar simulation with the half-explicit timestepping scheme on velocity level $\Delta t=1 \cdot 10^{-7} \mathrm{~s}, N=1000$.

notation of [2]:

$$
\begin{aligned}
& q_{i-1,0}=q_{i-1}, \\
& q_{i-1,1}=q_{i-1}+\Delta t_{i} v_{i-1}^{+} \text {, } \\
& v_{i-1,0}=v_{i-1}^{+} \text {, } \\
& v_{i-1,1}=v_{i-1}^{+}+\Delta t_{i} M_{i-1}^{-1}\left[h_{i-1}^{+}+W_{N_{i-1}} \lambda_{N_{i-1}}^{+}+W_{T_{i-1}} \lambda_{T_{i-1}}^{+}\right] \text {, } \\
& 0=\lambda_{N_{i-1}, \mathscr{I}_{1}^{i-1,0}}^{+}-\operatorname{proj}_{\mathbb{R}_{0}^{+}}\left(\lambda_{N_{i-1}, \mathscr{I}_{1}^{i-1,0}}^{+}-r W_{N, \mathscr{I}_{1}^{i-1,0}}^{T}\left(q_{i-1,1}\right) v_{i-1,1}\right), \\
& 0=\lambda_{T_{i-1}, \mathscr{I}_{1}^{i-1,0}}^{+}-\operatorname{proj}_{C_{T}\left(\lambda_{N_{i-1}, \mathscr{I}_{1}^{i-1,0}}^{+}\right)}\left(\lambda_{T_{i-1}, \mathscr{I}_{1}^{i-1,0}}^{+}-r W_{T, \mathscr{I}_{1}^{i-1,0}}^{T}\left(q_{i-1,1}\right) v_{i-1,1}\right)
\end{aligned}
$$




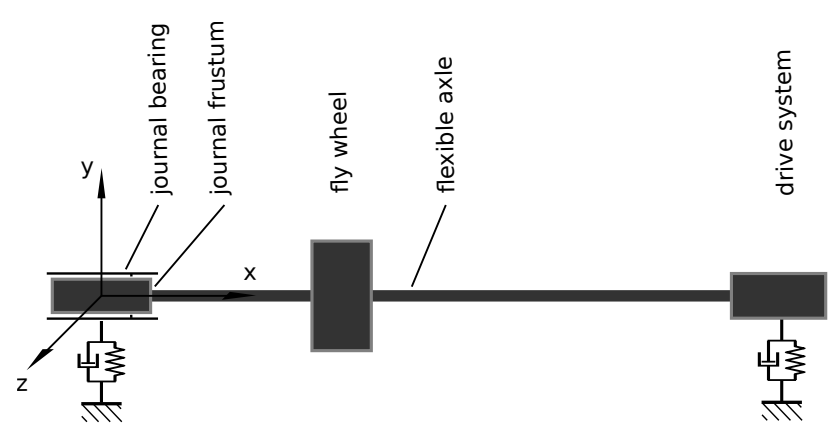

Figure 16: Model of a rotor test rig.

\begin{tabular}{llll}
\hline rotor axle & $l_{A}=0.59 \mathrm{~m}$ & journal bearing & $r_{J B}=1.01 \cdot 10^{-2} \mathrm{~m}$ \\
& $r_{A}=12.5 \cdot 10^{-3} \mathrm{~m}$ & & $m_{J B}=2.3 \mathrm{~kg}$ \\
& $\rho_{A}=7.85 \cdot 10^{3} \mathrm{~kg} / \mathrm{m}^{3}$ & & $x_{J B}=b_{J F} / 2$ \\
& $E_{A}=2.1 \cdot 10^{11} \mathrm{~N} / \mathrm{m}^{2}$ & & $\mu=0.01$ \\
& $G_{A}=0.81 \cdot 10^{11} \mathrm{~N} / \mathrm{m}^{2}$ & & $c_{J B}=10^{6} \mathrm{~N} / \mathrm{m}$ \\
& $d_{1_{A}}=601 / \mathrm{s}$ & $d_{J B}=10 \mathrm{Ns} / \mathrm{m}$ \\
& $d_{2_{A}}=0.001 \mathrm{kgm}^{2} / \mathrm{s}$ & & \\
\hline fly wheel & $m_{F}=4.98 \mathrm{~kg}$ & drive system & $T_{D}=100 \mathrm{Nm}$ for $0.05 \mathrm{~s}$ \\
& $J_{F}=0.01 \mathrm{kgm}^{2}$ & & $T_{F}=10 \mathrm{Nm}$ \\
& $x_{F}=1.8 \cdot 10^{-1} \mathrm{~m}$ & & $c_{D}=6 \cdot 10^{6} \mathrm{~N} / \mathrm{m}$ \\
\hline journal frustum & & & $d_{D}=10 \mathrm{Ns} / \mathrm{m}$ \\
& $r_{J F}=10^{-2} \mathrm{~m}$ & gravitation & $\gamma=9.81 \mathrm{~m} / \mathrm{s}^{2}$ \\
& $b_{J F}=19 \cdot 10^{-3} \mathrm{~m}$ & & \\
\hline
\end{tabular}

Table 6: Characteristics of the rotor example.

for the first stage unknowns $v_{i-1,1}, \lambda_{N_{i-1}}^{+}$and $\lambda_{T_{i-1}}^{+}$. For this interpretation, we have to change (A.2), e.g. to a forward Euler step. Then, we proceed:

$$
\begin{aligned}
q_{i} & =q_{i-1}+\frac{\Delta t_{i}}{2}\left\{v_{i-1,0}+v_{i-1,1}\right\} \\
v_{i}^{-} & =v_{i-1}^{+}+\frac{\Delta t_{i}}{2} M_{i-1}^{-1}\left[h_{i-1}^{+}+W_{N_{i-1}} \lambda_{N_{i-1}}^{+}+W_{T_{i-1}} \lambda_{T_{i-1}}^{+}\right]+\frac{\Delta t_{i}}{2} M_{i}^{-1}\left[h\left(q_{i}, v_{i-1,1}\right)+W_{N_{i}} \lambda_{N_{i}}^{-}+W_{T_{i}} \lambda_{T_{i}}^{-}\right] \\
0 & =\lambda_{N_{i}, \mathscr{I}_{1}^{i}}^{-}-\operatorname{proj}_{\mathbb{R}_{0}^{+}}\left(\lambda_{N_{i}, \mathscr{G}_{1}^{i}}^{-}-r W_{N_{i}, \mathscr{G}_{1}}^{T} v_{i}^{-}\right) \\
0 & =\lambda_{T_{i}, \mathscr{I}_{1}^{i}}^{-}-\operatorname{proj}_{C_{T}\left(\lambda_{N_{i}, \mathscr{O}_{1}^{i}}^{-}\right)}\left(\lambda_{T_{i}, \mathscr{I}_{1}^{i}}^{-}-r W_{T_{i}, \mathscr{G}_{1}^{T}}^{T} v_{i}^{-}\right) .
\end{aligned}
$$

The unknowns for the second stage are $v_{i}^{-}, \lambda_{N_{i}}^{-}$and $\lambda_{T_{i}}^{-}$. As an option, we can evaluate the impact equations without taking care, because they are also stated on velocity level and, as the case may be, automatically satisfied. Concerning efficient evaluations, however, we implement a robust procedure as shown in Fig. 6:

$$
\begin{aligned}
v_{i}^{+} & =v_{i}^{-}+M_{i}^{-1}\left[W_{N_{i}} \Lambda_{N_{i}}+W_{T_{i}} \Lambda_{T_{i}}\right], \\
0 & =\Lambda_{N_{i}, \mathscr{I}_{1}^{i}}-\operatorname{proj}_{\mathbb{R}_{0}^{+}}\left[\Lambda_{N_{i}}-r \dot{\bar{g}}_{N_{i}, \mathscr{I}_{1}^{i}}^{+}\right], \\
0 & =\Lambda_{T_{i}, \mathscr{I}_{1}^{i}}-\operatorname{proj}_{C_{T}\left(\Lambda_{N_{i}}\right)}\left[\Lambda_{T_{i}}-r \dot{\bar{g}}_{T_{i}, \mathscr{I}_{1}^{i}}^{+}\right] .
\end{aligned}
$$

The local post-impact velocities $\dot{\bar{g}}_{N_{i}, \mathscr{l}_{1}^{i}}^{+}$and $\dot{\bar{g}}_{T_{i}, \mathscr{l}_{1}^{i}}^{+}$are calculated according to (57). The unknowns are $v_{i}^{+}, \Lambda_{N_{i}}$ and $\Lambda_{T_{i}}$. 


\section{Appendix B. General half-explicit timestepping schemes on velocity level}

The "forecasting trapezoidal rule" is the easiest member of $D^{+}$or $D^{-}$timestepping schemes. Let $s$ be positive and represent the number of stages less one, i.e., $s=1$ for the "forecasting trapezoidal rule", as well as $l \in\{0, \ldots, s\}$ be an index iterating over the stages. If the following equations are solved simultaneously, $D^{-}$timestepping schemes can be interpreted as time-discontinuous Galerkin methods [10]:

$$
\begin{aligned}
q_{i-1, l} & =q_{i-1}+\Delta t_{i} \sum_{k} \beta_{i_{k}}\left(t_{i_{l}}\right) v_{i-1, k} \\
q_{i} & =q_{i-1}+\Delta t_{i} \sum_{k} \beta_{i_{k}} v_{i-1, k} \\
v_{i-1, l} & =v_{i-1}^{+}+\Delta t_{i} \sum_{k=0}^{s-1} \tilde{\beta}_{i_{k}}\left(t_{i_{l}}\right) M_{i-1, k}^{-1}\left[h_{i-1, k}+W_{i-1, k} \lambda_{i-1, k}\right] \\
v_{i}^{+} & =v_{i-1, s}+\Delta t_{i} \beta_{i_{s}}\left\{M_{i-1, s}^{-1}\left[h_{i-1, s}+W_{i-1, s} \lambda_{i-1, s}\right]-\sum_{k=0}^{s-1} \tilde{l}_{i_{k}}\left(t_{i}\right) M_{i-1, k}^{-1}\left[h_{i-1, k}+W_{i-1, k} \lambda_{i-1, k}\right]\right\}+M_{i}^{-1} W_{i} \Lambda_{i} .
\end{aligned}
$$

Thereby, the weights satisfy

$$
\beta_{i_{l}}=\frac{1}{\Delta t_{i}} \int_{I_{i}} l_{i_{l}} \mathrm{~d} t, \quad \beta_{i_{l}}\left(t^{*}\right):=\frac{1}{\Delta t_{i}} \int_{t_{i-1}}^{t^{*}} l_{i_{l}} \mathrm{~d} t
$$

with the Chebychev points $\left\{t_{i_{l}}\right\}_{l}$ and the classical pruned Lagrange polynomials

$$
l_{i_{l}}: I \rightarrow \mathbb{R}, \quad l_{i_{l}}(t):=\left\{\begin{array}{ll}
\prod_{j \neq l} \frac{t-t_{i_{j}}}{t_{i_{l}}-t_{i_{j}}}, & \text { for } t \in I_{i} \\
0, & \text { for } t \notin I_{i}
\end{array} .\right.
$$

The weights $\tilde{\beta}_{i_{l}}$ and the pruned Lagrange polynomials $\tilde{l}_{i_{l}}$ are defined accordingly for the interpolation of the first $s-1$ nodal values of $\dot{v}_{i-1, l}=M_{i-1, l}^{-1}\left[h_{i-1, l}+W_{i-1, l} \lambda_{i-1, l}\right](l \in\{0, \ldots, s-1\})$ :

$$
\begin{aligned}
& \tilde{\beta}_{i_{l}}=\frac{1}{\Delta t_{i}} \int_{I_{i}} \tilde{l}_{i_{l}} \mathrm{~d} t, \quad \tilde{\beta}_{i_{l}}\left(t^{*}\right):=\frac{1}{\Delta t_{i}} \int_{t_{i-1}}^{t^{*}} \tilde{l}_{i_{l}} \mathrm{~d} t, \\
& \tilde{l}_{i_{l}}: I \rightarrow \mathbb{R}, \quad \tilde{l}_{i_{l}}(t):= \begin{cases}\prod_{\substack{j \neq l \\
j \in\{0, \ldots, s-1\}}} \frac{t-t_{i_{j}}}{t_{i_{l}}-t_{i_{j}}}, & \text { for } t \in I_{i} \\
0, & \text { for } t \notin I_{i}\end{cases}
\end{aligned}
$$

The constraints for non-impulsive interactions $\left(q_{i-1, l}, v_{i-1, l}, \lambda_{i-1, l}, t_{i_{l}}\right) \in \mathscr{N}_{C}$ as well as for impulsive interactions $\left(q_{i}, v_{i}^{+}, \Lambda_{i}, t_{i}\right) \in \mathscr{N}_{I}$ may be calculated on velocity level. Because of the evaluation of the interpolation polynomials, the internal stages for position and velocity as well as their corresponding constraints are coupled, in general. For a half-explicit time-discretization, adaptations are necessary which may result in the loss of the interpretation as timediscontinuous Galerkin method.

The trapezoidal rule as a locally second order scheme suffices for most practical considerations and industrial simulations. Directly applying the half-explicit time-discretization with the notation of [2] as base-integration scheme 
results in the following generalization:

$$
\begin{aligned}
q_{i-1, l} & =q_{i-1}+\Delta t_{i} \sum_{k<l} a_{l k} v_{i-1, k}, \\
v_{i-1, l} & =v_{i-1}^{+}+\Delta t_{i} \sum_{k<l} a_{l k} M_{i-1, k}^{-1}\left[h_{i-1, k}+W_{N_{i-1}, k} \lambda_{N_{i-1}, k}+W_{T_{i-1}, k} \lambda_{T_{i-1}, k}\right], \\
0 & =\lambda_{N_{i-1}, l-1, \mathscr{I}_{1}^{i-1, l}}-\operatorname{proj}_{\mathbb{R}_{0}^{+}}\left(\lambda_{N_{i-1}, l-1, \mathscr{I}_{1}^{i-1, l}}-r W_{N, \mathscr{I}_{1}^{i-1, l}}^{T}\left(q_{i-1, l}\right) v_{i-1, l}\right), \\
0 & =\lambda_{T_{i-1}, l-1, \mathscr{I}_{1}^{i-1, l}}-\operatorname{proj}_{C_{T}\left(\lambda_{N_{i-1}, l-1, \mathscr{I}_{1}^{i-1, l}}\right)}\left(\lambda_{T_{i-1}, l-1, \mathscr{I}_{1}^{i-1, l}}-r W_{T, \mathscr{I}_{1}^{i-1, l}}^{T}\left(q_{i-1, l}\right) v_{i-1, l}\right) .
\end{aligned}
$$

This first set of equations works as follows. For step $l$, you know or easily calculate the current and all past positions; you also know all past velocities. The current velocity and the past Lagrange multiplier are unknown. That is why, you define the constraint in such a way that it is satisfied for the current velocities but the past Lagrange multiplier. Here, we have already included one improvement in comparison to Appendix A. As we know the current position, we can evaluate the current index set as already shown in Sect 6.2. At the end of the stage iteration, we know all position and velocity stages, but the last Lagrange multiplier stage is missing. That is why, we use the output equation to solve for it:

$$
\begin{aligned}
q_{i} & =q_{i-1}+\Delta t_{i} \sum_{k} b_{k} v_{i-1, k}, \\
v_{i}^{-} & =v_{i-1}^{+}+\Delta t_{i} \sum_{k} b_{k} M_{i-1, k}^{-1}\left[h_{i-1, k}+W_{N_{i-1}, k} \lambda_{N_{i-1}, k}+W_{T_{i-1}, k} \lambda_{T_{i-1}, k}\right], \\
0 & =\lambda_{N_{i-1}, s, \mathscr{I}_{1}^{i}}-\operatorname{proj}_{\mathbb{R}_{0}^{+}}\left(\lambda_{N_{i-1}, s, \mathscr{I}_{1}^{i}}-r W_{N_{i}, \mathscr{Y}_{1}^{i}}^{T} v_{i}^{-}\right) \\
0 & =\lambda_{T_{i-1}, s, \mathscr{I}_{1}^{i}}-\operatorname{proj}_{C_{T}\left(\lambda_{N_{i-1}, s, \mathscr{Y}_{1}^{i}}\right.}\left(\lambda_{T_{i-1}, s, \mathscr{I}_{1}^{i}}-r W_{T_{i}, \mathscr{I}_{1}}^{T} v_{i}^{-}\right) .
\end{aligned}
$$

The evaluations are meant with respect to $t_{i-1, l}=t_{i-1}+c_{l} \Delta t_{i}$. Coefficients $a_{l k}, b_{l}$ and $c_{l}$ can be defined as usual [7, 8]; in [2] a specific set of coefficients is given. Last, we correct impulses according to (A.11)-(A.13) if necessary.

\section{Acknowledgement}

JK gratefully acknowledges the UK's Engineering and Physical Sciences Research Council (EPSRC) for funding through a studentship.

\section{References}

[1] P. Alart, A. Curnier, A mixed formulation for frictional contact problems prone to Newton like solution methods, Comput Methods Appl Mech Engrg 92 (1991) 353 - 375.

[2] V. Brasey, Hem 5 user's guide, Tech. rep., Universite de Geneve, Suisse (1994).

[3] V. Brasey, Methodes demi-explicites pour equations differentielles algebraiques semi-explicites d'index 2, Ph.D. thesis, Universite de Geneve (1994).

[4] A. Murua, Phem 56 user's guide, Tech. rep., Universite de Geneve, Suisse (1995).

[5] A. Murua, Partitioned half-explicit Runge-Kutta methods for differential-algebraic systems of index 2, Computing 59 (1) (1997) 43-61

[6] M. Arnold, A. Murua, Non-stiff integrators for differential algebraic systems of index 2, Numerical Algorithms 19 (1-4) (1998) $25-41$.

[7] E. Hairer, G. Wanner, Solving Ordinary Differential Equations I: Nonstiff Problems, 2nd Edition, Vol. 8 of Springer series in computational mathematics, Springer, Berlin, 2009.

[8] E. Hairer, G. Wanner, Solving Ordinary Differential Equations II: Stiff and Differential-Algebraic-Problems, 2nd Edition, Vol. 14 of Springer series in computational mathematics, Springer, Berlin, 2010.

[9] J. J. Moreau, Numerical aspects of the sweeping process, Comput Methods Appl Mech Engrg 177 (1999) 329-349.

[10] T. Schindler, V. Acary, Timestepping schemes for nonsmooth dynamics based on discontinuous Galerkin methods: definition and outlook, Math Comput Simulat 95 (2013) 180-199.

[11] N. Newmark, A method for computation for structural dynamics, J Eng Mech 85 (EM 3) (1959) 67-94.

[12] H. Hilber, T. Hughes, R. Taylor, Improved numerical dissipation for time integration algorithms in structural dynamics., Earthquake Eng Struct Dynam 5 (3) (1977) 283-292. 
[13] J. Chung, G. Hulbert, A time integration algorithm for structural dynamics with improved numerical dissipation: the generalized- $\alpha$ method, J Appl Mech 60 (1993) 371-375.

[14] B. Simeon, Computational Flexible Multibody Dynamics, Springer, Berlin, 2013.

[15] O. Bauchau, Flexible Multibody Dynamics, Springer, Berlin, 2010.

[16] T. Laursen, Computational contact and impact mechanics, Springer, 2002.

[17] D. Doyen, A. Ern, S. Piperno, Time-integration schemes for the finite element dynamic Signorini problem, SIAM J Sci Comput 33 (2011) 223-249.

[18] J. Simo, N. Tarnow, The discrete energy-momentum method. conserving algorithms for nonlinear elastodynamics, Z Angew Math Phys 43 (5) (1992) 757-792.

[19] P. Deuflhard, R. Krause, S. Ertel, A contact-stabilized Newmark method for dynamical contact problems, Int J Numer Meth Eng 73 (9) (2008) 1274-1290.

[20] V. Acary, B. Brogliato, Numerical methods for nonsmooth dynamical systems : applications in mechanics and electronics, 1 st Edition, Vol. 35 of Lecture notes in applied and computational mechanics, Springer, Berlin, 2008.

[21] B. Wohlmuth, Variationally consistent discretization schemes and numerical algorithms for contact problems, Acta Numerica 20 (2011) $569-734$.

[22] R. Krause, M. Walloth, Presentation and comparison of selected algorithms for dynamic contact based on the Newmark scheme, Appl Numer Math 62 (10) (2012) $1393-1410$.

[23] P. Christensen, A. Klarbring, J.-S. Pang, N. Strömberg, Formulation and comparison of algorithms for frictional contact problems, Int J Numer Meth Eng 42 (1) (1998) 145-173.

[24] L. Qi, D. Sun, A survey of some nonsmooth equations and smoothing Newton methods, in: Progress in Optimization, Vol. 30 of Applied Optimization, Springer US, 1999, pp. 121-146.

[25] O. Brüls, V. Acary, A. Cardona, Simultaneous enforcement of constraints at position and velocity levels in the nonsmooth generalized- $\alpha$ scheme, Comput Methods Appl Mech Engrg 281 (2014) 131-161. doi:10.1016/j.cma.2014.07.025. URL http: / /hal.inria.fr/hal-01059823

[26] R. McLachlan, R. Quispel, Splitting methods, Acta Numerica 11 (2002) 341-434.

[27] T. Schindler, S. Rezaei, J. Kursawe, V. Acary, Half-explicit timestepping schemes on velocity level based on time-discontinuous Galerkin methods, Research Report RR-8823, INRIA, http: / / hal.inria.fr/hal-01078398 (2014).

[28] P. Wriggers, Computational contact mechanics, 1st Edition, Wiley, Chichester, 2002.

[29] R. Fetecau, J. Marsden, M. Ortiz, M. West, Nonsmooth Lagrangian mechanics and variational collision integrators, SIAM J Appl Dyn Syst 2 (2003) 381-416.

[30] B. Brogliato, Nonsmooth mechanics : models, dynamics and control, 2nd Edition, Communications and control engineering, Springer, London, 1999.

[31] C. Glocker, Set-valued force laws in rigid body dynamics : dynamics of non-smooth systems, 1st Edition, Vol. 1 of Lecture notes in applied and computational mechanics, Springer, Berlin, 2001.

[32] R. I. Leine, N. van de Wouw, Stability and convergence of mechanical systems with unilateral constraints, Vol. 36 of Lecture notes in applied and computational mechanics, Springer, Berlin, 2008.

[33] F. Pfeiffer, Mechanical system dynamics, corr. 2nd printing Edition, Vol. 40 of Lecture notes in applied and computational mechanics, Springer, Berlin, 2008.

[34] K. Johnson, Contact Mechanics, Cambridge University Press, New York, 2008.

[35] D. Stewart, Dynamics with inequalities, SIAM, Philadephia, 2011.

[36] P. Flores, R. Leine, C. Glocker, Modeling and analysis of planar rigid multibody systems with translational clearance joints based on the non-smooth dynamics approach, Multibody System Dynamics 23 (2010) 165-190.

[37] S. Schoeder, H. Ulbrich, T. Schindler, Discussion of the Gear-Gupta-Leimkuhler method for impacting mechanical systems, Multibody System Dynamics 31 (2014) 477-495.

[38] E. Eich-Soellner, C. Führer, Numerical methods in multibody dynamics, 2nd Edition, European Consortium for Mathematics in Industry, Teubner, Stuttgart, 1998.

[39] L. Paoli, M. Schatzman, A numerical scheme for impact problems i: the one-dimensional case, SIAM J Numer Anal 40 (2002) $702-733$.

[40] L. Paoli, M. Schatzman, A numerical scheme for impact problems ii: the multidimensional case, SIAM J Numer Anal 40 (2002) $734-768$.

[41] M. Jean, The nonsmooth contact dynamics method, Comput Methods Appl Mech Engrg 177 (1999) $235-257$.

[42] R. T. Rockafellar, Convex Analysis, 10th Edition, Princeton University Press, Princeton, 1997.

[43] T. Schindler, B. Nguyen, J. Trinkle, Understanding the difference between prox and complementarity formulations for simulation of systems with contact, in: IEEE/RSJ International Conference on Intelligent Robots and Systems, San Francisco, 25th-30th September 2011, 2011.

[44] V. Acary, Projected event-capturing time-stepping schemes for nonsmooth mechanical systems with unilateral contact and Coulomb's friction, Comput Methods Appl Mech Engrg 256 (2013) 224-250.

[45] C. Führer, B. Leimkuhler, Numerical solution of differential-algebraic equations for constrained mechanical motion, Numer Math 59 (1991) 55-69.

[46] M. Arnold, Numerical methods for simulation in applied dynamics, in: M. Arnold, W. Schiehlen (Eds.), Simulation Techniques for Applied Dynamics, no. 507 in CISM International Centre for Mechanical Sciences, Springer, Wien, 2009, pp. 191-246.

[47] C. W. Gear, B. Leimkuhler, G. Gupta, Automatic integration of Euler-Lagrange equations with constraints, J Comput Appl Math (1985) 77-90.

[48] E. Hairer, Symmetric projection methods for differential equations on manifolds, BIT 40 (2000) 726-734.

[49] Q. Chen, V. Acary, G. Virlez, O. Brüls, A nonsmooth generalized- $\alpha$ scheme for flexible multibody systems with unilateral constraints, Int J Numer Meth Eng 96 (8) (2013) 487-511.

[50] S. Rezaei, T. Schindler, Mixed timestepping schemes for nonsmooth mechanics with high frequency damping, Comput Struct submitted.

[51] D. Kaufman, D. Pai, Geometric numerical integration of inequality constrained, nonsmooth Hamiltonian systems, SIAM J Sci Comput 34 
(2012) 2670-2703.

[52] J. Marsden, M. West, Discrete mechanics and variational integrators, Acta Numerica 10 (2001) 357-514.

[53] D. Stewart, Rigid-body dynamics with friction and impact, SIAM Rev 42 (2000) 3-39.

[54] C. Studer, Numerics of unilateral contacts and friction : modeling and numerical time integration in non-smooth dynamics, Vol. 47 of Lecture notes in applied and computational mechanics, Springer, Berlin, 2009.

[55] R. Huber, H. Ulbrich, Higher order integration of non-smooth dynamical systems using parallel computed extrapolation methods based on time-stepping schemes, in: Proceedings of 1st Joint International Conference on Multibody System Dynamics, Lappeenranta, 25th-27th May 2010, 2010 .

[56] C. Lubich, Extrapolation integrators for constrained multibody systems, Impact of Computing in Science and Engineering 3 (3) (1991) 213-234.

[57] V. Acary, Higher order event capturing time-stepping schemes for nonsmooth multibody systems with unilateral constraints and impacts, Appl Numer Math 62 (10) (2012) 1259 - 1275.

[58] B. Esefeld, T. Schindler, H. Ulbrich, A coupling approach for the numerical integration of non-smooth multibody systems, in: Proceedings of the 4th Canadian Conference on Nonlinear Solid Mechanics, McGill University Montreal, Canada, 23rd-26th July 2013, 2013.

[59] P. Deuflhard, F. Bornemann, Scientific computing with ordinary differential equations, Vol. 42 of Texts in applied mathematics, Springer, New York, 2002

[60] V. Acary, F. Perignon, An introduction to Siconos, Tech. rep., INRIA (2007).

URL http://siconos.gforge.inria.fr

[61] MBSim - multi-body simulation software, GNU Lesser General Public License http://code.google.com/p/mbsim-env/.

[62] T. Schindler, M. Förg, M. Friedrich, M. Schneider, B. Esefeld, R. Huber, R. Zander, H. Ulbrich, Analysing dynamical phenomenons: introduction to MBSim, in: Proceedings of 1st Joint International Conference on Multibody System Dynamics, Lappeenranta, 25th-27th May 2010, 2010.

[63] L. Ginzinger, M. Sahinkaya, T. Schindler, H. Ulbrich, P. Keogh, Model-based condition monitoring of an auxiliary bearing following contact events, in: Proceedings of MOVIC 2010, August 17-20, 2010, Tokyo, Japan, 2010.

[64] M. Förg, Mehrkörpersysteme mit mengenwertigen Kraftgesetzen : Theorie und Numerik, als Manuskript gedruckt Edition, Vol. 411 of Fortschritt-Berichte VDI : Reihe 20, Rechnerunterstützte Verfahren, VDI Verlag, Düsseldorf, 2007. 